\title{
The influence of a porous, compliant layer with overlying discrete roughness elements as exhaust pipe wall on friction and heat transfer
}

\author{
C. Reurings $^{1}$ (D) S. Koussios ${ }^{1}$ - O. K. Bergsma ${ }^{1}$ - W.- P. Breugem ${ }^{2} \cdot{\text { K. } \text { Vergote }^{3} \cdot \text { L. Paeshuyse }^{3} \cdot \text { R. Benedictus }}^{1}$
}

Received: 29 March 2019 / Accepted: 9 March 2020 / Published online: 20 April 2020

(C) The Author(s) 2020

\begin{abstract}
The purpose of this work is to experimentally establish the combined influence on the flow and thermal resistance of an exhaust pipe wall formed by a porous, compliant layer with overlying discrete roughness elements exposed to the pulsating exhaust gas flow of a combustion engine. Through measuring the streamwise pressure drop over and radial temperature differences in different pipe samples for a range of flow states with different Reynolds numbers and nondimensional pulsation frequencies, the effects were discerned. The configurations of the sample walls covered a range of mesh pitches, compliant-layer densities, and compliant-layer compression ratios. The (non-sinusoidally) pulsating exhaust gas flow spanned the following range: $\operatorname{Re}_{b}\left(=u_{b} D / v_{b}\right)=1 \cdot 10^{4}-3 \cdot 10^{4}, T_{b}=500-800{ }^{\circ} \mathrm{C}, \omega^{+}\left(=\omega v_{b} / u_{\tau}^{2}\right)=0.003-$ 0.040. The friction factors were found to be effectively constant with Reynolds number and non-dimensional pulsation frequency while the variation with insulation density/compression was not significant. Additionally, for both mesh pitches, the measured friction factors were in line with those reported in literature for similar geometries with steady flow and solid walls. Together this indicates that neither compliance nor the pulsations in the exhaust gas flow significantly affect the friction for this configuration. Comparison of the samples based on the derived thermal resistance showed a similar influence of the fluid-wall interface as for the friction. Additionally a distinct influence of compression, independent of the insulation density, was observed that increases with increasing temperature. It was concluded that the increased resistance was due to additional radiation resistance because of fibre reorientation due to compression.
\end{abstract}

\section{List of Symbols}

\section{Latin Letter}

A Area $\left(\mathrm{m}^{2}\right)$

$A_{v} \quad$ Absorptance of the gas (-)

$c_{\mathrm{p}} \quad$ specific heat at constant pressure $\left(\mathrm{J} \mathrm{kg}^{-1} \mathrm{~K}^{-1}\right)$

$D \quad$ Diameter (m), without subscript: inner

$f_{D} \quad$ Darcy friction factor (-)

$k \quad$ Permeability $\left(\mathrm{m}^{2}\right)$

$L \quad$ Length of measurement segment (m)

C. Reurings

niels.reurings@gmail.com

1 Delft University of Technology, Aerospace Engineering, Kluyverweg 1, 2629HS Delft, The Netherlands

2 Delft University of Technology, J.M. Burgers Center for Fluid Dynamics, Leeghwaterstraat 21, 2628 CA Delft, The Netherlands

3 BOSAL ECS n.v., 20 Dellestraat, 3560 Lummen, Belgium $\dot{m} \quad$ mass flow rate $\left(\mathrm{kg} \mathrm{s}^{-1}\right)$

$N \quad$ Number of measurements in the set for a mean value (-)

$\mathrm{Nu} \quad$ Nusselt number (-)

$p \quad$ Pressure $\left(\mathrm{N} \mathrm{m}^{-2}\right)$

$p_{\text {mesh }} \quad$ Wire pitch of the mesh $(\mathrm{mm})$

Pr Prandtl number (-)

q Heat flux vector $\left(\mathrm{W} \mathrm{m}^{-2}\right)$

$q \quad$ Heat transfer rate $\left(\mathrm{J} \mathrm{s}^{-1}\right)$

$R \quad$ Radius (m)

Re Reynolds number (-)

$\operatorname{Re}_{K} \quad$ Permeability Reynolds number (-)

$S_{a_{b}} \quad$ Standard deviation of the error of type $a$ of the variable of interest from source $b$

$T \quad$ Temperature $\left({ }^{\circ} \mathrm{C}\right.$ or $\left.\mathrm{K}\right)$

$t \quad$ Time (s)

$t_{\text {ini }} \quad$ Initial thickness ( $\left.\mathrm{mm}\right)$

u Fluid velocity vector $\left(\mathrm{m} \mathrm{s}^{-1}\right)$

$u \quad$ Fluid velocity, streamwise direction $\left(\mathrm{m} \mathrm{s}^{-1}\right)$

$u_{\tau} \quad$ Friction velocity $\left(\mathrm{m} \mathrm{s}^{-1}\right)$ 


\section{$U_{a, b} \quad$ Uncertainty in variable $a$ at confidence $b$ (unit of a) \\ $V \quad$ Volume $\left(\mathrm{m}^{3}\right)$ \\ $v_{\mathrm{amb}} \quad$ Ambient convective air velocity $\left(\mathrm{m} \mathrm{s}^{-1}\right)$ \\ Wo Womersley number (-) \\ $x \quad$ Coordinate in streamwise direction (m)}

\section{Greek Letter}

$\beta \quad$ Coefficient of thermal expansion $\left(\mathrm{K}^{-1}\right)$

$\epsilon \quad$ Energy dissipation rate per unit mass $\left(\mathrm{m}^{2} \mathrm{~s}^{-3}\right)$

$\varepsilon \quad$ Strain (-)

$\lambda \quad$ Thermal conductivity $\left(\mathrm{W} \mathrm{m}^{-1} \mathrm{~K}^{-1}\right.$ )

$\mu \quad$ Dynamic viscosity $\left(\mathrm{N} \mathrm{s} \mathrm{m}^{-2}\right)$

$v \quad$ Kinematic viscosity $\left(\mathrm{m}^{2} \mathrm{~s}^{-1}\right)$

$\omega \quad$ Angular frequency of the pulsations $\left(\mathrm{rad} \mathrm{s}^{-1}\right)$

$\omega^{+} \quad$ Wall-normalized frequency (-)

$\Phi_{\text {inc }} \quad$ Rate of viscous dissipation for an incompressible fluid $\left(\mathrm{s}^{-2}\right)$

$\rho \quad$ Density $\left(\mathrm{kg} \mathrm{m}^{-3}\right)$

$\sigma \quad$ Stefan-Boltzmann constant $\left(\mathrm{W} \mathrm{m}^{-2} \mathrm{~K}^{-4}\right)$

$\tau_{w} \quad$ Wall shear stres $\left(\mathrm{N} \mathrm{m}^{-2}\right)$

$\chi \quad$ Volume compressibility $\left(\mathrm{m}^{2} \mathrm{~N}^{-1}\right)$

\section{Subscripts}

\begin{tabular}{|c|c|}
\hline $\mathrm{amb}$ & Ambient air property \\
\hline avg & Average \\
\hline$b$ & Bulk gas flow property \\
\hline$B_{a}$ & $\begin{array}{l}\text { Bias or systematic error of the variable from } \\
\text { source } a\end{array}$ \\
\hline cond & Conduction \\
\hline conv & Convection \\
\hline ini & Initial \\
\hline in & Inlet \\
\hline ins & Insulation \\
\hline out & Outlet \\
\hline$P_{a}$ & Random error of the variable from source $a$ \\
\hline 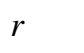 & Radial \\
\hline $\mathrm{rad}$ & Radiation \\
\hline$\theta$ & Circumferential \\
\hline
\end{tabular}

\section{Introduction}

To date, the conventional steel exhaust system has contributed to control of the mass of non-electric road vehicles and thus their emissions. The exhaust system mass has generally reduced through a combination of improved design capabilities and steel compositions. However under the increasingly strict emission standards, such as those for passenger vehicles in the EU [14], there is a need for further mass reduction.

One of the options for exhaust systems would be to look for alternatives with increased specific performance, but those are not abundant. The use of a high-strength and highdensity material such as steel is not driven by mechanical requirements, but mostly by the demanding combination of durability requirements. Just the peak temperatures, of close to $1000^{\circ} \mathrm{C}$, hugely limit the number of alternative materials.

Switching to multi-material solutions however, could enable a larger set of solutions with higher specific performance than the conventional steel system. More specifically: lining the exhaust with a low-mass and low-conductivity layer could thermally enable a much larger material set for the duct itself.

Understanding the effect of the addition of such a layer on the flow resistance and on the thermal resistance between the gas and duct under the non-steady exhaust gas flow of an internal combustion engine is crucial to this concept.

Friction and convective heat transfer are both fluid-wall interaction phenomena and thus dependent on the flow and wall state. They are macroscopic quantities and, as such, not all subtlety of the complex turbulent flow is of equal influence. It proved possible to establish relative simple correlations for friction and heat transfer in the canonical case of steady, turbulent pipe flow with smooth, solid walls and a small temperature difference [20]. For turbulent flows with more complicated boundary properties and geometries establishing the friction and heat transfer rates has proved more difficult.

Because of its relevance to cooling systems and other industrial flow systems, friction and heat transfer for turbulent pipe flow has been of interest for at least close to a century $[11,19]$. In many cases, the heat transfer, in the form of the Nusselt number, is correlated to the friction factor and consequently these aspects are often investigated together.

Bhatti \& Shah [5] compared many of the correlations that were proposed over time for the friction factor and Nusselt number for steady turbulent flow in pipes with walls of homogeneous (sand-grain, $k$-type) roughness. In those cases the main factors of influence are the relative roughness height, the level of turbulence (Reynolds number) and the Prandtl number of the fluid.

For boundaries with discrete roughness, the spacing and geometry of the roughness elements also play a role. As a result, no general expressions for the friction factor for such cases exist and one has to resort to datasets such as ESDU 79014 [13] or individual articles describing particular configurations [33]. In principle, the same holds for the heat transfer to walls with discrete roughness elements, although Norris [30] introduced a general albeit coarse expression that covers quite a range of geometries.

In sinusoidally pulsating turbulent pipe flow, the effect on the friction factor depends on the amplitude and the frequency of the oscillation that is superimposed on the mean flow. The trend in the results of Lodahl et al. shows that in smooth pipes the friction is unaffected as long as the mean velocity is larger than the oscillating velocity amplitude, 
i.e. in the current-dominated regime. Wave-dominated flow, on the other hand, can substantially alter the mean friction magnitude [26]. Furthermore, the results from Bhaganagar suggest that also for rough walls current-dominated pulsating flow does not affect the friction, unless there is a specific length coupling with the roughness geometry [4].

To the authors knowledge, no studies have refuted the general correlation between friction and heat transfer for pulsating flow in smooth pipes. Analogous to friction, substantial increases in heat transfer have been reported for wave-dominated flow: for sinusoidal pulsations by Ludlow et al. [27] and for pulse-combustors see Meng et al. [28]. For current-dominated flow, no investigations towards the effect of sinusoidal pulsations on heat transfer were found. The trend of the results of Ludlow et al. towards that regime, however, indicates little deviation from the steady flow values. In that sense, the increase in heat transfer that Wendland reports for tailpipes of car-mounted exhaust systems is remarkable but not necessarily conflicting given their curvature [37].

If, contrary to all of the above studies, the out-ofplane stiffness of flow boundary is relatively low and its deformation and potential interaction with the flow no longer negligible, then it can be considered compliant. Although the effect of boundary-compliance on the delay of transition to turbulence is well-established, for turbulent flow both friction increases and decreases have been reported without consensus on the interaction [38]. The reported effect on friction was however generally small (< $10 \%$ ) for smooth walls. No work on rough walls or its effect on heat transfer was found.

A porous wall can lose part of its wall-blocking and no-slip properties, depending on its relative permeability, resulting in mixing between the wall and bulk fluid with substantially increased friction and heat transfer as a result [7, 24]. General expressions for these increases are not possible due to the dependence on geometry, porosity and relative permeability [8].

The configuration of the lined exhaust wall in this study has several of these factors in combination and therefore covers new ground. Its porous and compliant wall surface is kept in place using a wire mesh that classifies as discrete roughness and it is exposed to the pulsating exhaust gas flow. Given the uncertainty in the influence of compliance and the non-sinusoidal nature of the pulsating flow, the friction and heat transfer of this configuration is already worth investigating, let alone because of the potential interaction between the three mentioned effects.

Next to the effect of the wall on the fluid-wall interaction, there is also the thermal resistance inside the porous layer that is of interest. Assuming the permeability of the interface is indeed negligible, then the thermal resistance of fibrous insulation is in principle well understood for the density range of interest.
Between the solid and the gas phase in the insulation, there are four different modes of heat transfer possible: gas conduction, solid conduction, natural convection and radiation. Not all of these modes are equally relevant. At the densities and temperatures under investigation, for instance, natural convection and solid conduction are negligible. The main heat transfer mechanisms are thus radiation and gas conduction; especially the former is a complex mechanism: it depends highly on the optical properties of the fibres (as a function of wavelength) and on the geometry (fibre distribution and size) $[9,10,39]$.

The two dominant mechanisms, radiation and gas conduction, have different sensitivities to insulation compression. Gas conduction is at these high porosities effectively independent of the solid fraction, because the mean free path is substantially smaller than the mean distance between fibres $(\mathrm{Kn} \leq 0.01)$ [9]. The effect of compression on the radiation resistance however depends on the resulting fibre distribution, because the fibres could rotate or translate or both with different results. If substantial rotation occurs, then a higher thermal resistance can be achieved with the same insulation mass. For mass critical applications such as these, it is therefore worthwhile to investigate the effect of compression on the thermal resistance.

The purpose of this work is to establish the yet undescribed combined influence of an exhaust pipe wall formed by a compliant layer with overlying discrete roughness elements exposed to pulsating turbulent flow on the friction factor and their combined thermal resistance and, at the same time, assess the effect of compression of the compliant layer on the thermal resistance.

This study examines these effects experimentally using a series of instrumented samples placed downstream of a combustion engine in a controlled environment. Through measuring the pressure drop and temperature difference over these samples for a range of flow states with different Reynolds numbers and non-dimensional pulsation frequencies, the effects could be discerned. The wall configurations of these samples cover a range of mesh pitches, compliantlayer densities, and compliant-layer compression ratios.

This articles is organized as follows: Section 2 describes the samples, setup, and equipment, and the employed procedure and data processing. Subsequently, Section 3 shows the obtained results and compares the trends to those from literature. Finally, Section 4 provides the conclusions.

\section{Materials and methods}

The essence of the method is as follows: determine the friction and heat loss rate using, respectively, the static pressure and bulk gas temperature drop over samples that represent lined exhaust sections. A schematic representation 
Table 1 General measurement and analysis overview

\begin{tabular}{|c|c|c|c|c|}
\hline \multirow{3}{*}{$\begin{array}{l}\text { Aspect: } \\
\text { measurement }\end{array}$} & \multicolumn{4}{|l|}{ Post-processing } \\
\hline & \multicolumn{2}{|l|}{ Reference sample } & \multicolumn{2}{|l|}{ Lined samples } \\
\hline & Input & Output & Input & Output \\
\hline $\begin{array}{l}\text { Friction: sample } \\
\text { pressure drop }\end{array}$ & $\begin{array}{l}\text { section pressure } \\
\text { drop (theory) }\end{array}$ & $\begin{array}{l}\text { connector } \\
\text { pressure drop }\end{array}$ & $\begin{array}{l}\text { connector } \\
\text { pressure drop }\end{array}$ & $\begin{array}{l}\text { section } \\
\text { pressure drop }\end{array}$ \\
\hline $\begin{array}{l}\text { Heat loss: section } \\
\text { gas temperature } \\
\text { drop }\end{array}$ & $\dot{m}, c_{\mathrm{p}}$ & $\begin{array}{l}\text { section heat } \\
\text { loss }\end{array}$ & $\dot{m}, c_{\mathrm{p}}$ & $\begin{array}{l}\text { section heat } \\
\text { loss }\end{array}$ \\
\hline $\begin{array}{l}\text { Heat loss: external } \\
\text { heat loss model }\end{array}$ & $\begin{array}{l}T_{\mathrm{amb}}, v_{\mathrm{amb}}, \\
T_{\text {shell }}\end{array}$ & $\begin{array}{l}\text { section heat } \\
\text { loss }\end{array}$ & $\begin{array}{l}T_{\mathrm{amb}}, v_{\mathrm{amb}}, \\
T_{\text {shell }}\end{array}$ & $\begin{array}{l}\text { section heat } \\
\text { loss }\end{array}$ \\
\hline
\end{tabular}

The samples are an assembly of a cylindrical test section with a connector at each end of the test setup is provided in Fig. 3 and a general overview of the different measurements is provided in Table 1. The computed friction factors were subsequently compared with reference values from literature to differentiate between various contributions. The subsequent sections detail the aspects of the samples, measurements setup, procedure and processing of the results.

\subsection{Samples}

In order to have a flow state relevant to exhaust systems, the sample's geometry was similar to a section of this envisioned application: a straight pipe lined with the multimaterial wall under investigation. Such a axisymmetric sample is more difficult to manufacture than a flat one, but ideally offers the advantage of straightforward onedimensional heat transfer.

In the flow direction, the samples consisted of a lined section with connectors at its up- and downstream end. The central section of the sample was a 1-metre long polymer cylindrical shell internally lined with a porous ceramic fibre layer and a silica fabric. These were kept in place through a stainless steel mesh. In Fig. 1 a schematic representation of the cross-section of the sample wall is given. The separate components of the sample are detailed in the paragraphs below.

Firstly, the square mesh was made of stainless steel wires of $1.0 \mathrm{~mm}$ diameter placed at a pitch of $11.0 \mathrm{~mm}$. The wires were not woven but welded; the axial wires were radially on the inside of the circumferential wires. The average outer diameter of the circumferential rings was $59.8 \mathrm{~mm}$. At both ends, there was a steel pipe section welded around the mesh extending beyond it, ensuring alignment with the inner tube of the connector. For some of the samples, the streamwise pitch was increased to $23 \mathrm{~mm}$ by removing the wire segments between the axial wires of every second circumferential ring. Small parts of the removed rings thus remained at the welds, keeping the fabric at the same distance. For one sample, the mesh was replaced altogether by a $1.5 \mathrm{~mm}$ thick solid steel tube with an outer diameter of $60.0 \mathrm{~mm}$.

Secondly, covering the outside of this mesh was a twillweave silica fabric of $0.44 \mathrm{~mm}$ thick. To assess the possibility of interaction, the absolute permeability of the fabric was measured according to ASTM F778 [1] and found to be $k$ $=2 \times 10^{-11} \mathrm{~m}^{2}$. According to Hahn et al. [16], the effective permeability of a wall in turbulent flow can be classified using the ratio of the effective pore diameter of the wall
Fig. 1 Schematic representation of a portion of the sample cross-section with components indicated (left), sample 18-1H-1 before adhesion of the connectors (middle), and schematic representation of a sample during testing with the shell flanges vertical and a gas thermocouple mount on one side (right)
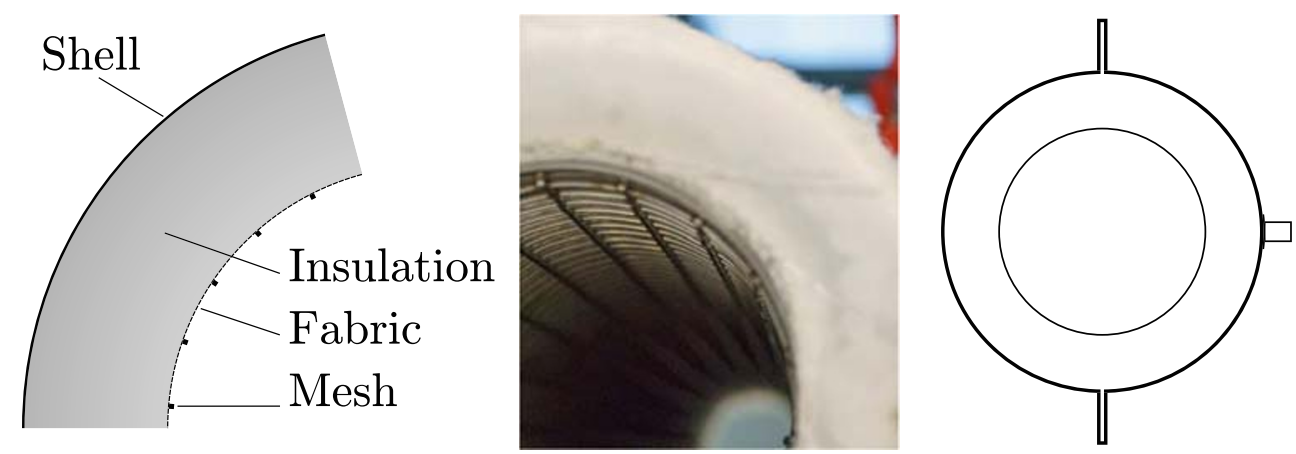
$\left(k^{1 / 2}\right)$ over the wall length unit $v_{b} / u_{\tau}$, where $v_{b}$ is the kinematic viscosity of the fluid and $u_{\tau}$ the friction velocity: $u_{\tau}=\sqrt{\tau_{w} / \rho_{b}}$.

Thirdly, the fibrous ceramic material that formed the insulation layer had porosity exceeding 0.9 , a mean fibre diameter of several $\mu \mathrm{m}$ and consisted primarily of silica. The material was in blanket-form and two different bulk densities were used: 96 and $128 \mathrm{~kg} \mathrm{~m}^{-3}$. For the density range used in this study, the fibre volume fraction was between 3 and $4 \%$. To study the potential influence of the insulation material on the friction and heat transfer, different densities with different amounts of compression were tested. By radially compressing an amount of material to fit the space for the insulation, the density of this layer was increased. Not only could this alter the fibre distribution, it could also result in a reaction force pushing against its domain boundary, altering the compliance of the wall. The different sample insulation densities can be found in Table 2 together with corresponding amount of compression.

Each sample had several layers of either of the two densities, but because the layers do not slide relative to each other and tend to buckle locally when bent or compressed, the assembly proved arduous. The general result was consistently applied insulation around the circumference except at the longitudinal seam, where gaps were observed for one or more layers locally along the length. The insulation seam was always positioned at one of the shell flanges. In some cases the rotational symmetry of the heat transfer was affected by this locally reduced density and thermal resistance; shell temperatures of up to $30^{\circ} \mathrm{C}$ higher than the mean have been observed near the flange at high engine loads. Hence an additional method to derive the heat transfer rate was employed based on the external thermal resistance.

The limited compressibility of the material and its discrete thickness allowed just two configurations with compressed insulation, but it is nevertheless important to know

Table 2 Sample overview: mesh pitch, initial (pre-assembly) insulation thickness and density, sample insulation density, average radial strain and average volume change

\begin{tabular}{lllllll}
\hline Sample & $\begin{array}{l}p_{\text {mesh }} \\
\mathrm{mm}\end{array}$ & $\begin{array}{l}t_{\text {ini }} \\
\mathrm{mm}\end{array}$ & $\begin{array}{l}\rho_{\text {ini }} \\
\mathrm{kg} \mathrm{m}^{-3}\end{array}$ & $\begin{array}{l}\rho_{\text {sample }} \\
\mathrm{kg} \mathrm{m}^{-3}\end{array}$ & $\begin{array}{l}\varepsilon_{r, \text { avg }} \\
-\end{array}$ & $\begin{array}{l}\Delta V / V \\
-\end{array}$ \\
\hline 128-16-HS & solid & 16 & 128 & 129 & $-0,01$ & $-0,01$ \\
128-16-H & 11 & 16 & 128 & 129 & $-0,01$ & $-0,01$ \\
128-16-HR & 23 & 16 & 128 & 129 & $-0,01$ & $-0,01$ \\
128-18-H & 11 & 18 & 128 & 149 & $-0,12$ & $-0,14$ \\
96-20-H & 11 & 20 & 96 & 127 & $-0,20$ & $-0,25$ \\
\hline
\end{tabular}

The initial properties are according to factory specification and sample insulation compression properties follow from the Lamé solution with the initial density as input, see Fig. 2 the resulting material density. The strains corresponding to radial compression of a cylinder under internal and external pressure are described by the Lamé solution [35]. If the plane-strain solution is assumed and the Poisson's ratio taken as zero, then this solution can approximate the strains corresponding to the compression of a network of randomly oriented fibres, the result of which is depicted in Fig. 2. The radial strain is largest at the inner radius and in general much larger than the circumferential strain, causing the fibres to reorient towards the plane perpendicular to the radial direction. Compression thus makes the insulation anisotropic with a fibre orientation bias towards the circumferential direction, perpendicular to thermal radiation coming from the fluid-interface. Also depicted in Fig. 2 is an estimate of the volume change, which indicates that the volume (and thus density) change is practically homogeneous over the radius.

Fourthly, the duct, or shell, had an average outer diameter of $93.3 \mathrm{~mm}$ and a thickness of $1.0 \mathrm{~mm}$. It was made of two halves to allow for installation of the inner layers. The $15 \mathrm{~mm}$-wide longitudinal flanges of these two halves were subsequently bonded using a temperature resistant adhesive, see the right hand side of Fig. 1 for an impression. The thermal conductivity of the shell material is of the order of $1 \mathrm{~W} \mathrm{~m}^{-1} \mathrm{~K}^{-1}$ which, combined with the limited connector temperature near the shell, makes axial heat conduction along the shell negligible compared to the radial heat transfer.

The adhesively bonded connectors provided the thermal and mechanical connection of the central part to the other elements of the setup. Essentially, these connectors consist of two concentric cylinders of the same diameters as the sample mesh and shell. At one end of the connector, the outer cylinder meets the inner one and there is a flange to attach it to other setup elements. The outer wall thus provides the mechanical connection to the polymer shell. The sole purpose of the inner wall is to guide the flow to the lined section; it has a helical structure allowing its segments to slide axially along each other, accommodating thermal expansion differences. This inner structure forms a flow boundary that is not completely smooth, because the gap between segments forms a rectangular groove.

To distinguish the effect of the mesh geometry, insulation density and compression on the friction and heat transfer separately, several configurations were manufactured and tested. An overview of the different samples and their designation is given in Table 2. The flow interface was varied between a smooth solid tube to a mesh with pitches of 11 and $23 \mathrm{~mm}$, with a base insulation density of $128 \mathrm{~kg}$ $\mathrm{m}^{-3}$ without compression. The insulation compression and density were varied in two different ways in combination with the mesh pitch of $11 \mathrm{~mm}$. First, to obtain the same insulation density as $128-16-\mathrm{H}$, but with substantial insulation compression and second, to have a considerably 
Fig. 2 Estimation of the radial, $\epsilon_{r}$, and circumferential strain, $\epsilon_{\theta}$, corresponding to the radial compression of $20-\mathrm{mm}$ of insulation down to roughly 16 $\mathrm{mm}$ for a material with zero

Poisson's ratio and plane strain. The third curve is the corresponding volume change: $\Delta V / V \approx\left(1+\epsilon_{r}\right)\left(1+\epsilon_{\theta}\right)-1$

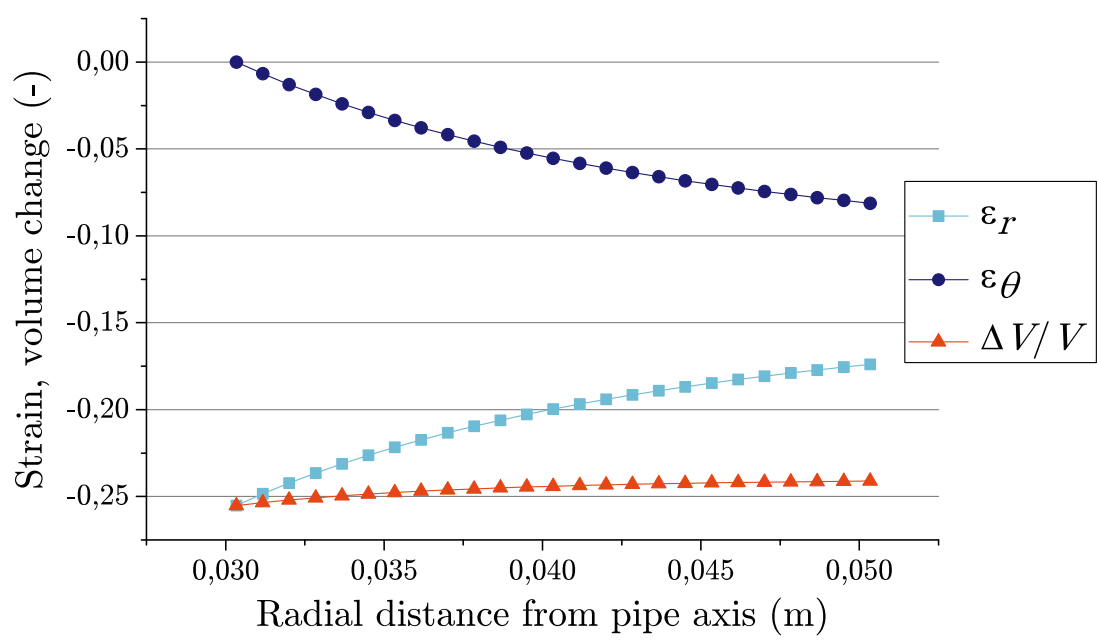

higher density. Each configuration except the one lined with the solid tube was manufactured and tested in duplicate to assess the consistency and scatter.

The sample with the solid steel inner wall serves as benchmark because friction and convective heat transfer in hydraulically smooth pipes with turbulent flow is well understood. Its wall lining is also definitively impermeable.

\subsection{Setup}

In the setup, each of the described samples was placed downstream of a dynamometer-mounted gasoline V6 engine. The first elements downstream of the merging point of the two engine manifolds were a flexible exhaust joint and a straight pipe section with several sensor ports, together spanning a streamwise length of about $750 \mathrm{~mm}$. The sample in turn was mounted to this pipe through its connector with the shell flanges aligned vertically. A schematic representation of the setup is given in Fig. 3; the sample orientation is shown schematically on the right side of Fig. 1.

Coming out of the sample through the downstream connector, the exhaust gasses passed through a heat exchanger and a mass flow sensor and finally ended up in a large silencing vessel that exits into the atmosphere. The heat exchanger was placed downstream of the sample to reduce the exhaust gas temperature to within the validity range of the mass flow sensor. Because the cooling water flow was actively controlled, the gas temperature could be preset and it was maintained at about $110^{\circ} \mathrm{C}$.

No pressure ports were placed in the lined section to prevent influencing the flow or temperature field. This meant that the static pressure ports had to be placed a few centimetres up- and downstream of the connectors. Both ports were connected to a single transducer to measure only the pressure difference.

The thermocouples for the gas temperature were positioned in the flow using dedicated steel mounts that were adhered to the shell after having drilled the required holes. They assured a similar depth for each measurement and air-tight placement. All three mounts were on the same locations on the circumference: at the top of a shell half, see the right hand side of Fig. 1. The resulting flow penetration depth of the thermocouple sheath tip relative to the fabric was 38 $\mathrm{mm}$. The longitudinal locations of the three mounts were at

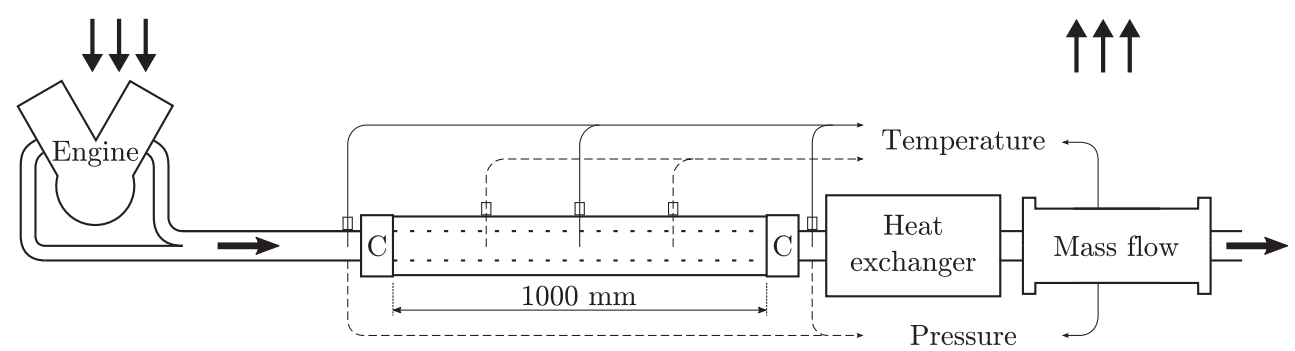

Fig. 3 Schematic representation of the test setup with, from left to right, V6-engine, manifolds and interface pipe, sample with connectors ('C'), heat exchanger, and mass flow sensor. Also indicated are the locations of gas temperature and pressure measurement: differential measurement (dashed line) and point measurement (solid line). The test cell ventilation entry and exit locations are indicated by the two sets of three arrows 
250,500 and $750 \mathrm{~mm}$ from the upstream edge of the 1000 $\mathrm{mm}$ shell. The instrumented length of the sample was thus half of its length: $500 \mathrm{~mm}$ and it was thus this length that was used in all relevant calculations.

As shown in Fig. 3, the middle of the three gas thermocouples was used to measure the mean gas temperature and the other two for the temperature drop along the sample. Each thermocouple had three hot junctions along its exposed length, yet only those closest to the tip were used in the subsequent calculations because their temperatures proved most consistent. The only exception to that was the use of the temperatures from the three hot junctions in the central thermocouple to determine the radial gradient in Appendix B.

Attempts were made to measure the temperature near at the fabric inside the sample, but the radial temperature gradient was too large to measure accurately using thermocouples. Other in situ temperature measurement techniques were not available or not suitable.

Active ventilation ensured that the test cell air temperature was constant to about $1{ }^{\circ} \mathrm{C}$ for a certain test stage. The air circuit was an open one, meaning that the air was taken from outside. Given the time-span of the test, this meant that the inlet temperature was practically constant and in combination with the thermal mass of the test cell, this resulted in a stable ambient air temperature.

The inlet of the air flow is in the ceiling above the engine and the outlet also in the ceiling but above the mass flow sensor, see Fig. 3. It was estimated that near the sample the air flow direction was both downward and axial. To prevent interference of the shell flanges with the external flow, the samples were mounted with the flanges vertical and thus aligned with the stagnation points of a cylinder in cross-flow.

Because of the vertical component of the air flow, the forced convection-dominated thermal resistance outside of the sample is not exactly rotationally symmetric. For consistent comparison, only shell temperatures measured at locations the furthest away from the flanges were used in the subsequent calculations. Looking at the right side of Fig. 1, this means they were at the same height as the gas thermocouple brackets or opposite. Lengthwise, these shell thermocouples spanned the same range as the gas thermocouples. In detail, the hot junctions of the two-wire thermocouples were taped to the shell in the middle between the gas thermocouple mounts and three more were placed exactly opposite these mounts, thus on the other side of the shell.

\subsection{Equipment}

The engine used to generate the turbulent gas flow is a 3.2 litre, naturally aspirated, four-stroke, petrol V6 (General Motors Company, USA) with a transversely mounted exhaust system. Each cylinder bank has its own manifold with integrated catalytic convertor. The exits of these two manifolds meet in a Y-intersection and downstream of that the flow can be classified as non-sinusoidal, non-reversing and having a Womersley number (Wo $=R \sqrt{\omega / v_{b}}$ ) of at least 70 [6].

A hydraulic dynamometer (FroudeHofmann, now Froude, USA) was used to regulate the engine speed and power. It regulates the engine rotational speed with an accuracy of $1 \mathrm{RPM}$, which corresponds to an accuracy of $0.04 \%$ for the speed of 2600 RPM that was used in all results reported here. The engine output was controlled through the throttle and this was reflected in the torque, which was measured at $1 \mathrm{Nm}$ accuracy, which corresponds to 0.6 - 5 $\%$, for the applied range of the engine loads.

The mass flow sensors employed were of the calibrated pitot type, requiring pressure transducers for the static and dynamic pressure. Two different diameter flow sensors were used to have a dynamic pressure of sufficient magnitude relative to the employed transducer accuracy. Both were calibrated to an accuracy of $1 \%$.

All pressures (static pressure difference over the sample and the absolute dynamic and static pressure of the mass flow sensor) were measured using a DMQ*-DT pressure transducer ( $\mu$ mess $\mathrm{GmbH}$, Germany) with an error of 0.2 mbar. An anomaly was observed in the static pressure drop readings for all samples at Reynolds numbers below $2 \cdot 10^{4}$, probably because of interaction between the transducer internal averaging and the non-steady flow. Consequently, only the pressure drop values obtained at Reynolds numbers of roughly $2 \cdot 10^{4}$ and higher were used to determine the friction factors.

To determine the connector friction factor and to prove that the anomaly was sensor related, the test with 128-16HS was performed using a pressure sensor that did not show anomalous behaviour at $\operatorname{Re}<2 \cdot 10^{4}$, namely a GE DPI 705 (General Electric Company, US) with a full scale error of 0.07 mbar. The result of that test is shown in Fig. 6 .

All exhaust gas temperature measurements were performed using sheathed, special tolerance K-type thermocouples (Thermo Electric, the Netherlands), yet in terms of geometry and data acquisition there were differences. All the exhaust gas flow temperature measurement inside the sample was performed using thermocouples with an Inconel outer sheath of $3.2 \mathrm{~mm}$ in diameter and $0.5 \mathrm{~mm}$ in thickness and housing three individual sheathed thermocouples with their junctions at a $10-\mathrm{mm}$ interval from the outer sheath tip. Between the up- and downstream sample thermocouple, the junction pairs were wired for differential measurement, ${ }^{1}$

\footnotetext{
${ }^{1}$ In hindsight this did not improve the accuracy by much because it introduced an additional error through the non-linear temperaturevoltage relation of the K-type on top of the lower thermocouple tolerance of $1.1^{\circ} \mathrm{C}$.
} 
as indicated in Fig. 3. The data acquisition for these thermocouples was performed using a Keithley Integra 2701 with a 7708 switch card (Tektronix, USA). For the differential temperatures, a $0{ }^{\circ} \mathrm{C}$ simulated cold junction was used resulting in a data-acquisition error of $0.2^{\circ} \mathrm{C}$; for the absolute temperatures, the automatic cold junction compensation resulted in a $1.0^{\circ} \mathrm{C}$ error. Over the range of gas temperatures tested, the manufacturer calibrated tolerance in the absolute temperature measurement for the employed K-type thermocouples ranged from 2.2 to $3.2^{\circ} \mathrm{C}$. An analysis of the total temperature uncertainty can be found in Appendix A.

A set of regular manufacturer tolerance K-type wire thermocouples was used to measure the shell temperature on both sides of the sample and also near the flanges. Their wires were spot welded to form the hot junctions. Using the FroudeHofmann system, the data acquisition error was 1.0 ${ }^{\circ} \mathrm{C}$. Furthermore, at the shell temperatures encountered the individual thermocouple tolerance was $2.2^{\circ} \mathrm{C}$.

The air temperature in the cell was measured directly underneath the ventilation inlet at the engine intake, and at the ceiling away from the air stream; in both cases using an RTD with an accuracy of about $1.0^{\circ} \mathrm{C}$. The atmospheric pressure and humidity were also measured.

All measured quantities were recorded at sampling rate of $1 \mathrm{~Hz}$, with the exception of the exhaust gas temperature measurement inside the sample which was at $0.5 \mathrm{~Hz}$.

\subsection{Procedure}

For all data presented here, the same set of thermocouples, the same engine and the same test cell was used. Furthermore, the gas temperature sensors were placed in the same streamwise order.

Depending on whether the small or large diameter mass flow sensor was in place, the corresponding engine sequence that was programmed in the dynamometer software was run. Each sequence consisted of a set of stages with varying engine loads but always the same engine speed (2600 RPM). Effectively the engine load, and consequently the mass flow rate and gas temperature, was increased with successive stages according to the maximum dictated by the mass flow sensor. Having finished the first sequence, the mass flow sensor was swapped for the other diameter one and the other sequence was started without changing anything else.

Combining both sequences, six unique stages were run with the mean gas temperature and Reynolds number varying between 600 and $800{ }^{\circ} \mathrm{C}$ and $1 \cdot 10^{4}$ and $3 \cdot 10^{4}$, respectively. Figure 4 shows the bulk gas velocity and gas temperature as a function of the engine torque, which is representative for all samples. Even though the engine speed, and thus its firing frequency, was kept constant, all other fluid and flow properties did change with engine load. As a consequence, the wall-normalized frequency $\omega^{+}(=$ $\omega v_{b} / \bar{u}_{\tau}^{2}$ ) also varied with engine load, more specifically, for the sample of Fig. 4 it decreased from 0.040 to 0.003 with the engine torque increasing over the sketched range. The overscore on the friction velocity indicates the time-mean value.

Several checks for consistency between and within the sequences were performed for each sample. Firstly, both sequences could be compared because the first stage was the same. Secondly, within each sequence the last stage had the same engine settings as the first. Repeating a stage with the same sample on the same day resulted in gas and shell temperatures that were within a few degrees.

The common first stage that subjects the samples to the largest gas temperature change, was used to determine the duration needed for convergence towards thermal equilibrium. Initial tests showed that duration of this stage
Fig. 4 The bulk gas velocity (diamonds, left axis) and gas temperature (circles, right axis, relative to ambient) as a function of engine torque at constant engine speed for sample 18-H-1

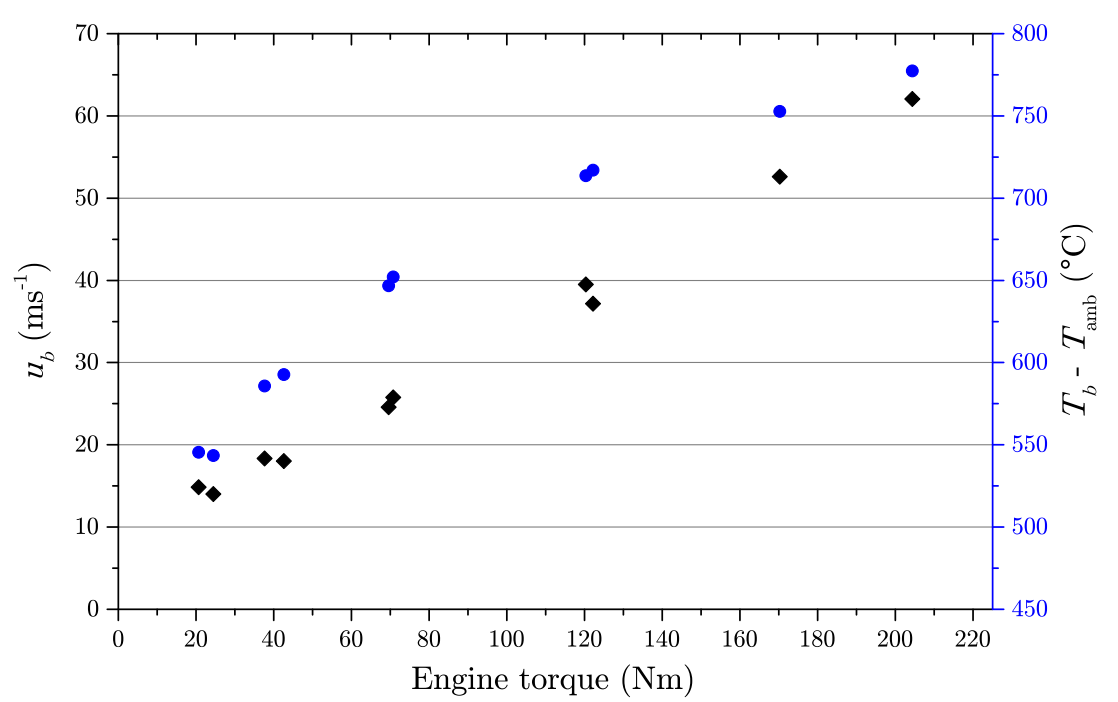


of 20 minutes is sufficient to have a shell temperature that differs less than $1.0{ }^{\circ} \mathrm{C}$ from its exponential asymptotic value (obtained using a least-squares fit). This stage duration was adopted for all stages in the engine sequence, because the temperature increments become smaller for the successive stages.

The raw data also shows that there was scatter in the engine output in terms of torque and gas temperature; for this reason all measured quantities were time-averaged over the last minute of a stage in the post-processing. The average standard deviations of the gas and shell temperature over the last 60 seconds of each stage of both sequences were 1.0 and $0.6^{\circ} \mathrm{C}$, respectively.

\subsection{Data processing and analysis}

The assumptions behind and methods used to approximate or derive the thermophysical, friction and heat transfer properties are defined in this section. First, the thermal equilibrium of the gas thermocouple is treated to provide an indication of the temperature difference between it and the gas. Second, the thermophysical properties of the flue gas, which differ from dry air, are discussed. Third, the employed diameter for the flow domain of the samples with mesh lining is treated together with the average flow velocity. Fourth, the friction factor calculation is outlined. Fifth, the axial heat balance of the flue gas is presented together with the resulting heat loss rate. Because of inconsistency observed in this heat loss rate between similar samples, an alternative heat loss rate estimation was also employed. The sixth subsection treats this alternative heat loss rate method. It includes the electrical analogy for radial one-dimensional heat transfer to relate the two methods and it also treats the thermal resistance model of the environment that it is based on.

\subsubsection{Thermocouple deviation}

The equilibrium temperature of a sheathed thermocouple tip in a gas flow results predominantly from the balance between forced convection and radiation and it could thus deviate significantly from the gas temperature depending on the influence of the radiation. If radiation contributes substantially, then the extent of this deviation depends on the infrared transparency of the gas flow, because that defines whether the radiative heat exchange is with the wall or the gas.

In order to estimate the temperature difference between that of the sheathed thermocouples and the gas itself, the heat balance of the thermocouple was modelled one-dimensionally. Details and results of this model can be found in Appendix B. In the end, a temperature-dependent correction was applied to the thermocouple gas temperatures, see Fig. 13.

\subsubsection{Thermophysical properties}

The combustion process that takes place in the cylinders of the engine alters the composition of the exhaust gas and thus the difference in properties compared to dry air was determined. It was assumed that the exhaust gas composition, used in all subsequent property calculations, could be approximated by the result of the idealized stoichiometric reaction between humid air and gasoline (in the form of octane, $\mathrm{C}_{8} \mathrm{H}_{18}$ ).

The molar fraction of water in the humid air was calculated using the method of Appendix A.1 of Picard et al. [32] with the measured air pressure and humidity as input. Its density can be obtained using the ideal gas law because at the temperatures and pressures under consideration, the resulting flue gas qualifies as an ideal gas [22].

The specific heat capacity, dynamic viscosity and the thermal conductivity were all obtained using correlations for the temperature-dependent gas component properties and a suitable mixing rule $[22,23] .^{2}$ As a result, the specific heat of the exhaust gas obtained in this manner is about $12 \%$ higher than that of dry air at the same temperature and pressure. This is the largest relative deviation from dry air for all mentioned thermophysical properties.

In all subsequent calculations the properties of the exhaust gas flow are based on these expressions.

\subsubsection{Diameter, flow velocity and Reynolds number}

For both Reynolds number and friction factor computation, a diameter describing the flow channel is needed. For all the samples with a mesh interface, the diameter is not trivial. For these samples all subsequent computations that involve the diameter of the flow domain, $D$, the outer diameter of the mesh of $59.8 \mathrm{~mm}$, i.e. the interface with the fabric, was employed.

The time-mean bulk flow velocity was based on the mass flow rate as follows:

$u_{b}=\frac{\dot{m}}{\rho_{b} \pi\left(\frac{D}{2}\right)^{2}}$

where $D$ is the diameter of the flow duct and $\rho_{b}$ is the average density of the exhaust gas. Similarly for the mean bulk flow Reynolds number:

$\operatorname{Re}_{b}=u_{b} D / v_{b}=\frac{4 \dot{m}}{\rho_{b} v_{b} \pi D}$

where $v_{b}$ is the kinematic viscosity of the bulk fluid.

The combined uncertainty in the mass flow rate is dominant for both the bulk velocity as the bulk Reynolds number and as a result the uncertainty of all three is in the range of 2-4\%, depending on the engine load.

\footnotetext{
${ }^{2}$ In D3.1 Table 8 the $D$-coefficient of $\mathrm{H}_{2} \mathrm{O}$ should have a minus sign.
} 


\subsubsection{Friction factor}

The friction factor definition used in this study is that of Darcy-Weisbach:

$f_{D}=\frac{\Delta p}{L} \frac{D}{\frac{1}{2} \rho_{b} u_{b}^{2}}$

where $\Delta p$ is the pressure drop over streamwise length $L$ in a pipe with diameter $D$.

To obtain the friction factor of the lined sections of the samples of interest, the contribution from the sample connectors has to be subtracted from the total pressure drop, see Fig. 3. The pressure drop of these connectors can be obtained using the reference sample, because the friction factor of a smooth pipe is well-covered in literature. The employed expression for the friction factor of a smooth pipe in steady turbulent flow is the Techo et al. [20] explicit form of the Prandtl, Karman and Nikuradse correlation. This expression for non-pulsating flow should be applicable as exhaust gas flow is current dominated and according to Lodahl et al. the friction factor for smooth pipes is then not affected by the pulsations [26]. The fact that the pulsation is not sinusoidal should not matter much in the current-dominated regime.

Additionally, the pressure drop due to the gas flow thermocouples was determined by running the experiment with and without them installed.

The friction factors obtained for the connectors and thermocouples can subsequently be used to establish the pressure drop of the lined section of the other samples because these components are the same.

The flow entering the sample through the first connector should be close to or completely fully developed. The development length in steady flow for the turbulent Reynolds numbers at hand ranges from 14.0 to 17.1 hydraulic diameters according to the expression of Zhi-qing (see Table 4.12 of Bhatti and Shah) and Kirmse showed that similar lengths hold for the pressure gradient in pulsating flows [5, 21]. The circular section leading up to the sample connector has a length of about $12.5 \mathrm{D}$ and ahead of that, the duct is still circular and mostly straight for another few diameters but it does include the Y-intersection of the two manifold exits. The flow must thus at least be close to fully developed.

In all samples there is a sudden change in wall geometry from the connector to the centre section and vice versa, and that has a certain effect on the measured total pressure drop. Siuru and Logan have shown that for the transition from smooth to rough in turbulent flow, the transition length is a few roughness heights, which is negligible compared to the total length of the connector [34]. Even if the transition length for the change from rough to smooth is a few diameters, then that is still relatively short compared to the total connector and lined section lengths. In the samples with the lined section with discrete roughness, the transition is from rough to rough, which is a smaller difference than from smooth to rough and the effect is then also assumed small.

\subsubsection{Heat loss rate}

Another aspect of the flow is its temperature drop and that can, in certain cases, be related to its heat loss. If we apply the first law of thermodynamics in rate-form to an axial segment of the fluid domain inside the sample and then assume steady-state conditions, no changes in latent energy, no thermal or mechanical energy generation, an ideal gas, and both negligible pressure variation and viscous dissipation, then it reduces to the well-known form [2]:

$q=\dot{m} c_{\mathrm{p}}\left(T_{b, \text { in }}-T_{b, \text { out }}\right)$

where $q$ is the heat loss rate of the fluid over the instrumented length of the sample in $\mathrm{W}, \dot{m}$ is the mass flow rate in $\mathrm{kg} \mathrm{s}^{-1}, c_{\mathrm{p}}$ is the specific heat for constant pressure in $\mathrm{J} \mathrm{kg}^{-1} \mathrm{~K}^{-1}$, and $T_{b, \text { in }}$ and $T_{b, \text { out }}$ represent the bulk temperature in ${ }^{\circ} \mathrm{C}$ (or $\mathrm{K}$ ) of the fluid entering and exiting the segment, respectively.

If furthermore the axial conductivity is assumed negligible, then only the radial heat flux remains. Equation 4 can thus be used to relate the heat loss through the wall of the test section to the bulk temperature drop of the gas. The uncertainty of this rate under the stated assumptions is treated in Appendix A.

Most of the stated assumptions are easily verified, but given the relatively large temperature, velocity and friction, a negligible contribution of viscous dissipation is not obvious. Consequently, an estimate of its magnitude was made in Appendix $\mathrm{C}$. The result of this estimate is that at the highest engine load the temperature change through viscous dissipation could exceed $10 \%$ of the measured temperature drop. At that flow state, the true heat loss is thus somewhat larger than what is obtained from Eq. 4 because part of the temperature drop was compensated by viscous heating.

\subsubsection{Alternative heat loss rate method}

Figure 5 shows the electrical analogy of the one-dimensional radial heat flow in the lined section of the sample. The resistance elements in this analogy are the temperaturedependent thermal equivalents (unit: $\mathrm{K} \mathrm{W}^{-1}$ ) of electrical resistance [2]. The thermal resistance of the outer polymer shell and that of the steel inner tube in case of sample 128-16-HS are assumed negligible and thus excluded.

Because the test environment of the samples was always the same, a model of the external thermal resistance can provide an additional measure of the heat loss based on just 


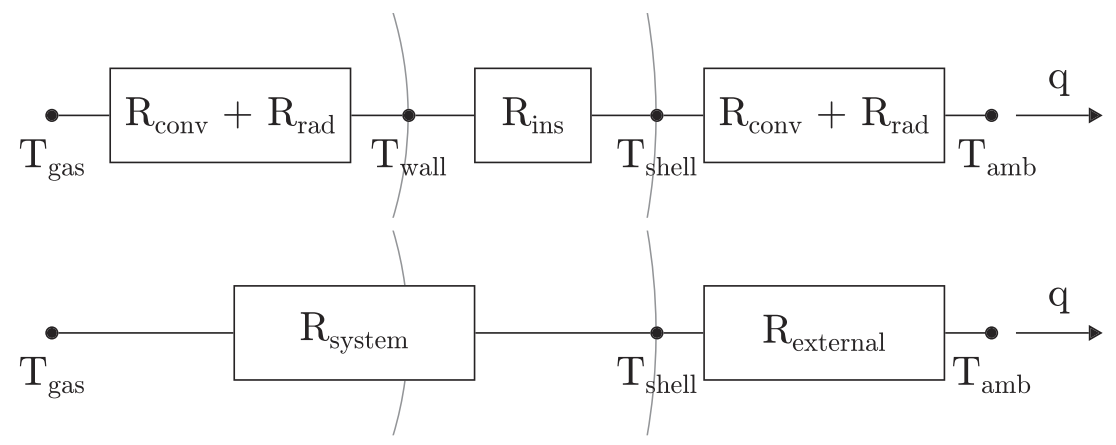

Fig. 5 Schematic representation of the resistance elements in the electrical analogy of the radial heat transfer. Between the gas and wall there is the combination of convection and radiation, within the insulation the dominant mechanisms are lumped into one effective resistance

the temperature of the shell and that of the environment. The main heat transfer mechanisms, and thus those that constitute this model, are forced convection and radiation.

Convective heat loss is often described in terms of the Nusselt number:

$q_{\text {conv }}=\mathrm{Nu} \lambda_{\mathrm{amb}} / D_{\text {shell }}\left(T_{\text {shell }}-T_{\mathrm{amb}}\right) A_{\text {shell }}$

where $\lambda$ is the thermal conductivity of the air and the subscripts shell and amb indicate the sample shell and environment, respectively.

The employed expression for the Nusselt number of a cylinder in turbulent cross-flow was that of Churchill and Bernstein [20]:

$\mathrm{Nu}=0.3+\frac{0.62 \operatorname{Re}_{D \text { shell }}^{1 / 2} \operatorname{Pr}^{1 / 3}}{\left[1+\left(\frac{0.4}{\mathrm{Pr}}\right)^{2 / 3}\right]^{1 / 4}}\left[1+\left(\frac{\operatorname{Re}_{D \text { shell }}}{282000}\right)^{5 / 8}\right]^{4 / 5}$

to which the Gnielinski correction for non-isothermal fluid properties [15] was applied because use of the film temperature was deemed inaccurate given the sizeable temperature difference and where $\operatorname{Pr}\left(=\mu c_{\mathrm{p}} / \lambda\right)$ is the Prandtl number.

To model the radiation it was assumed that the area of the sample (cylinder) was much smaller than that of the enclosing space, allowing a simple expression for radiative heat exchange [18]:

$q_{\mathrm{rad}}=\varepsilon_{\text {shell }} \sigma \pi D_{\text {shell }} L\left(T_{\text {shell }}^{4}-T_{\mathrm{amb}}^{4}\right)$

where $\sigma$ is the Stefan-Boltzmann constant. The emissivity value of 0.93 for glass-epoxy from Berlin et al. [3] was employed.

The only unknown in the model that could not be reasonably estimated is the ventilation velocity. It was thus obtained for each sample by correlating the heat loss from the gas flow temperature drop through a least-squares fit to the heat loss from the external model, where the
$R_{\text {ins }}$ and outside the sample, the external resistance is a combination of forced convection and radiation. In the absence of $T_{\text {wall }}$, the thermal resistances between the gas and shell can be lumped together into one system resistance $R_{\text {system }}$ (bottom representation)

latter is a function of the ventilation velocity based on expressions Eqs. 6 and 7. For these fits, the stages with an estimated viscous dissipation contribution exceeding 5\% were excluded. The smallest of the obtained ventilation velocities was employed in the model, ensuring the smallest influence of insulation flaws on the result.

\section{Results \& discussion}

\subsection{Friction}

To obtain the friction factors that are of interest, namely those of the lined sections of the samples presented in Table 2, requires the friction factors of the other components. This is treated first, in line with Table 1 . For the reference sample the pressure drop corresponding to the smooth section was subtracted from the measured total back pressure of the sample, both with and without gas thermocouples, to determine the contributions of the connectors and gas thermocouples separately. The resulting Darcy-Weisbach friction factor $f_{D}$ of the connectors is shown in Fig. 6 as a function of Reynolds number. Also shown in this figure is the smooth-wall friction factor according to the explicit form of Techo et al. used to compute the smooth section pressure drop.

The connector friction is effectively constant over the tested range of Reynolds numbers and non-dimensional frequencies $\omega^{+}$. The magnitude of the connector friction factor is furthermore consistent with the Reynolds numberindependent value obtained from the ESDU 79014 data set [13] for steady flow: $f_{D}=0.06$, where the helical elements of the connector were represented as rectangular ribs. The equivalent sand-grain roughness of the connector was obtained using the diameter and Nikuradse's expression for the relative roughness as a function of the friction factor [20]: $\mathrm{e} / \mathrm{D}=0.031$ and $k_{s}=1.7 \mathrm{~mm}$. This is larger than the estimated groove depth of $1 \mathrm{~mm}$, but within expectation 
Fig. 6 Subtraction of the back pressure of the smooth section of 128-16-HS-1 from the total static pressure drop without the gas thermocouples in place allowed determination of the friction factor due to the connectors. Both are shown here with corresponding error bars

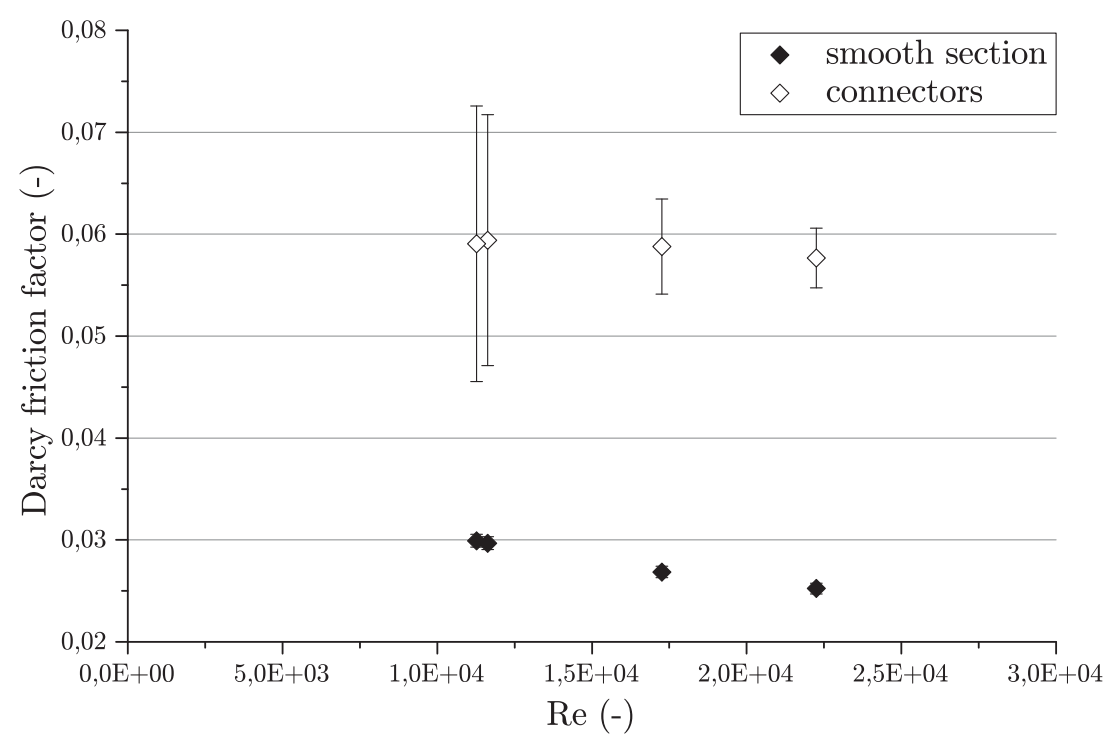

because of the larger flow influence of discrete roughness elements. This is also reflected in the roughness regime classification using the Moody diagram [20]: the connectors are in the transition regime for the three lowest Reynolds numbers and fully rough for the highest one. The curve of constant relative roughness is, even in the transitional regime, relatively flat over the Reynolds number range of interest, which is in line with the lack of Reynolds number dependence in Fig. 6.

The current results for the connectors indicate that the conclusion of Bhaganagar [4] based on direct numerical simulation, namely that the friction of a surface with periodic roughness is unaffected by pulsating flow as long as there is no specific length coupling between the oscillation amplitude and the geometry of the roughness, also holds for discrete roughness of larger size and spacing.
The effect of the gas thermocouples on the friction is much smaller than that of the connectors and also constant with Reynolds number.

Having the contribution to the pressure drop of the other components, now the friction of the lined sections of the other samples can be determined. The contribution of the connectors and the thermocouples to the total pressure drop was smaller than the pressure drop over the lined section, even for the samples with the largest mesh pitch. The average lined-section friction factors, including error bars, of all sample types are graphically compared in Fig. 7 and a few things stand out.

Firstly, similar to the connector friction factor in Fig. 6, the friction factors of the samples listed in Table 2 are, within the error, constant with Reynolds number and with the simultaneously varying non-dimensional frequency.
Fig. 7 Darcy-Weisbach friction factors of the lined sections of samples of all types with corresponding error bars. Multiple bars indicate multiple samples of the same type. The labels are according to Table 2

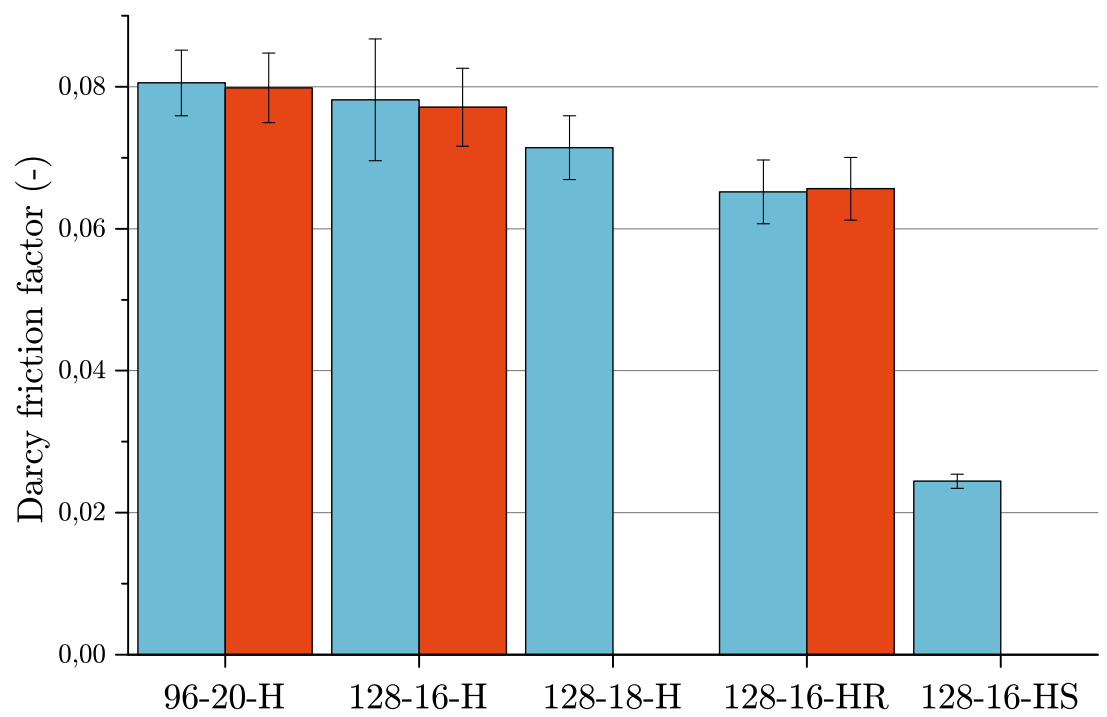


Within the accuracy of these tests, the discrete roughness section thus seems to be equally insensitive to the pulsations as the connector.

Secondly, the porous compliant wall seems to have little influence. Replacing the solid smooth wall with a wiremesh at the same insulation density (128-16-H) results in a tripled friction factor, which, in terms of magnitude, is in the range of that observed in literature for solid walls in steady turbulent flow lined with similar roughness types. For instance, using the methodology of ESDU 79014 [13] for semi-circular ribs and circular threads results in $f_{D}=0.070 \pm 0.014$ and $f_{D}=0.094 \pm 0.014$, respectively. Similarly, Sheriff and Gumley [33] report $f_{D}=0.084$ for a wall lined with a $1-\mathrm{mm}$ wire at a pitch of $10 \mathrm{~mm}$. These sources lacked the longitudinal wires to form a mesh like the sample has, but their influence should be smaller than that of the circumferential ones. The equivalent sand-grain roughness size of $3.5 \mathrm{~mm}$ for $128-16-\mathrm{H}$ is substantially larger than the mesh wire diameter of $1.0 \mathrm{~mm}$ and confirms the larger (form) drag of discrete roughness elements compared to closely-spaced ones. Together this suggests that the discrete roughness could, as for the connectors, be the dominant factor.

Thirdly, the friction of samples with an increased circumferential wire pitch is also not substantially affected by the pulsating flow or the underlying compliant, porous wall. The friction factor of $128-16-\mathrm{HR}$ is $16 \%$ lower than with the smaller pitch and same insulation density. This decrease is in line with that observed in ESDU 79014 for circular thread walls with the same pitch increase: $-16 \%$. This also points to the discrete roughness as the dominant factor.

Fourthly, there is a small decrease in friction factor visible along the left three sample types in Fig. 7 with increasing insulation density. This could be due to the porous, compliant wall material underneath the roughness elements. Direct interaction between the fluid in the insulation and in the flow is not expected because the ratio between the effective pore diameter of the liner and the wall length unit, the permeability Reynolds number $\operatorname{Re}_{K}=k^{1 / 2} u_{\tau} / v_{b}$, is about 0.1 for the employed fabric which is too small for the wall to be effectively permeable according to Breugem et al. [7]. Another mechanism that could affect the friction is the compliance of the wall, but only in between the mesh wires and with the right coupling with the normalized frequency. Since both the frequency and the wall properties (through the temperature) vary over the test sequence, it could explain part of the intrasample variation in friction between test stages, but not a consistent difference between samples. A last mechanism that could affect the friction is the curvature of the fabric in between the mesh wires: through a decrease in the volume in between the drag could decrease. This would be expected largest for the samples with the largest compression, 96$20-\mathrm{H}$, but because those report the highest friction factor this mechanism cannot be responsible. Either way, for the current range of flow and wall properties the influence of the compliant wall is small compared to that of the roughness, but otherwise indecisive.

\subsection{Thermal resistance}

Without feasible means to accurately measure the wall temperature in this configuration without influencing the wall-fluid interface or the heat transfer, the convective heat transfer cannot be analysed independently. That does however not affect the ability to compare the different samples in terms of the combined thermal resistance of the
Fig. 8 Thermal resistance of the system, i.e. between gas and outer shell, for the samples with different flow interfaces but the same insulation density as a function of the temperature difference between the gas and ambient $\left(T_{b}-T_{\mathrm{amb}}\right)$

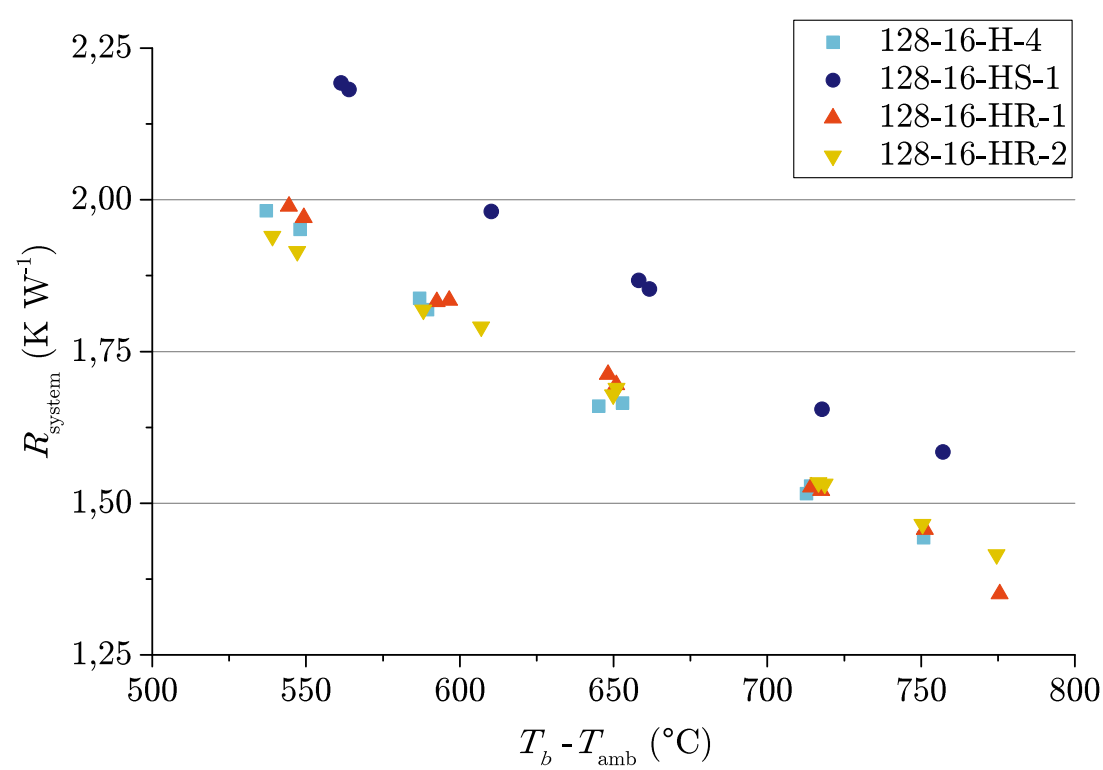


insulation and the wall-fluid interface, $R_{\text {system }}$, see Fig. 5 . For all samples, the total heat loss used to compute the resistance with was obtained from the earlier described external resistance model using the local shell temperature. The total thermal resistance of different configurations is compared and correlations to the friction, insulation density, and compression differences are studied, see for instance Fig. 8.

As mentioned in the introduction, the interest lies in both the thermal resistance between the fluid and the wall and the thermal resistance of the insulation. As such, it is worthwhile to estimate the relative contributions to $R_{\text {system. This }}$ is possible for the reference sample as the system resistance is known just as for the other samples and its fluid-to-wall resistance can be estimated based on available literature.

The resistance between fluid and wall comprises a convective and radiative component and these are well described for the smooth ducts of 128-16-HS. Its convective resistance was calculated using the Nusselt number expression of Gnielinski for a smooth solid circular ducts (Table 8.3 of Karwa [20]) and the net radiative thermal energy exchange rate between a gas and wall was computed according to [36]:

$q=A \sigma \frac{\varepsilon_{\text {wall }}}{1-\left(1-\varepsilon_{\text {wall }}\right)\left(1-A_{v}\right)}\left(\varepsilon_{b} T_{b}^{4}-A_{v} T_{\text {wall }}^{4}\right)$

where $\varepsilon_{b}$ and $A_{v}$ are the emissivity and absorptance of the gas mixture and $\varepsilon_{\text {wall }}$ represents the emissivity of the wall. Of the gas components only water and carbon dioxide were taken into account as a function of their partial pressure, the temperature and equivalent layer thickness. The results show that the contribution of the radiative heat transfer cannot be ignored, because its contribution to the total heat exchange from gas to wall varies between $11 \%$ and $24 \%$.
The relative contributions of the fluid-to-wall and insulation resistance can be compared through the ratio of the resulting thermal resistances. At low engine load the ratio of $R_{\text {ins }} /\left(R_{\text {conv }}+R_{\text {rad }}\right)$ is 6 , meaning that the insulation is dominant. As both the insulation and fluidto-wall thermal resistance of 128-16-HS-1 depend on the engine load and decrease a similar amount in absolute terms, the ratio increases to 13 at the highest engine load, meaning the insulation resistance is even more dominant.

Starting with the system heat transfer of the samples with the same density and compression as the solid-walled one. The effect of the altered flow interface on the heat transfer is clear, both are shown in Fig. 8. The wire-mesh sample has a thermal resistance that is $13 \%$ less at the lowest engine load (and temperature difference) and $11 \%$ at the highest. This difference is substantially smaller than the factor three difference in friction and this is in part due to the relatively small contribution of the fluid-to-wall resistance and in part because the convective resistance for the discrete roughness sample will be smaller due to the increased friction. Also, the increase of the mesh pitch has no distinguishable effect on the system thermal resistance. The data points of 12816- $\mathrm{H}$ and $-\mathrm{HR}$ in Fig. 8 clearly overlap. The decrease in friction and the corresponding increase in convective resistance is simply too small to affect the system resistance significantly. In short, for the samples with the same insulation density and compression the trends for the heat transfer are consistent with those for friction when taking into account the relative magnitude of the fluid-to-wall resistance and this consistency substantiates the idea from the friction analysis that there are no substantial effects due to the interaction between the pulsating flow, the compliant wall and the wire roughness.
Fig. 9 System thermal resistance of the benchmark sample, 128-16-H-4, and those with the same density but substantial insulation compression: 96-20-H

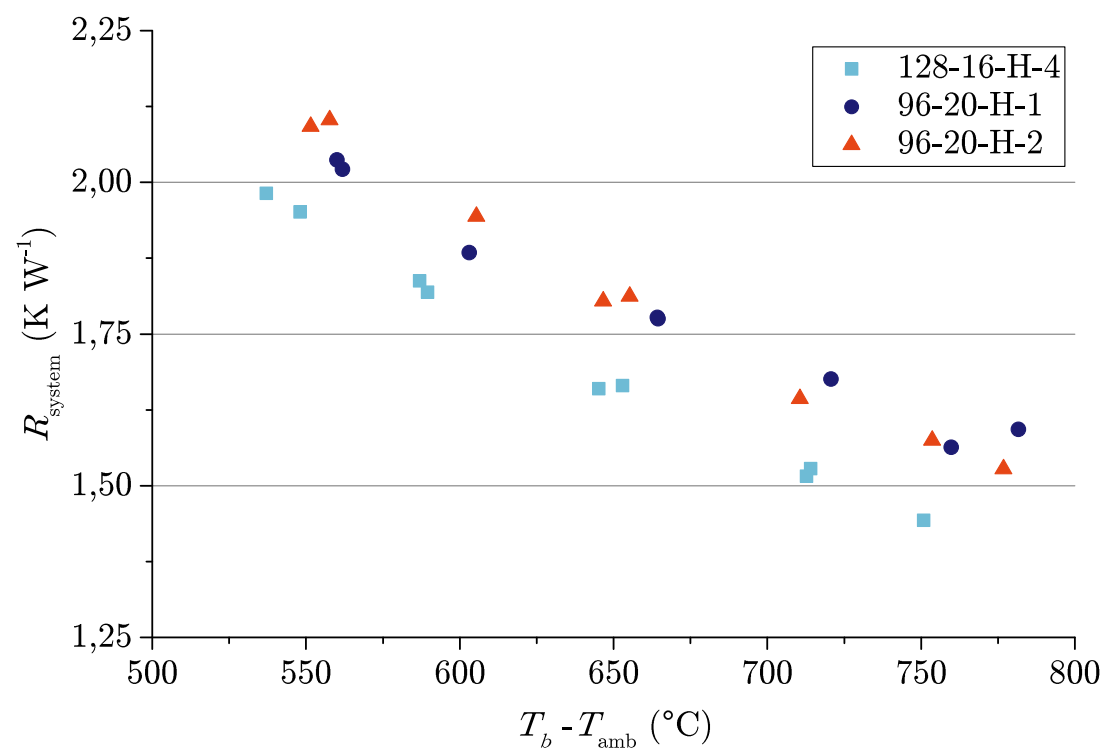


On the other hand, the samples with a similar insulation density as $128-16-\mathrm{H}$, but with significant compression (9620-H) have a distinctly larger thermal resistance, see Fig. 9. The average thermal resistance difference of the 96-20 samples with 128-16-H increases from about $7 \%$ larger at low engine loads to $11 \%$ larger at the high engine loads. As the flow interface is similar in terms of geometry, which was confirmed by a comparable friction factor, the difference must be due to the insulation compression. Also, as gas conduction cannot explain the difference because it is insensitive at these densities, the effect has to be due to radiation.

Indeed, the radiation resistance can be related to the compression of the insulation. Firstly, the gas side insulation temperatures are high enough for radiation to contribute substantially to the total heat transfer. Secondly, inherent to the mechanism, the distribution and orientation of the fibres is of direct influence on radiation heat transfer [25]. The nett unidirectional compression can cause alignment towards the direction perpendicular to it, i.e. perpendicular to the radial (and thus radiation) direction, increasing the radiation resistance compared to the non-compressed sample. As the contribution of radiation to the total heat transfer increases with temperature, so does the difference in $R_{\text {system }}$ between the two samples. Hence, the slight divergence of the resistance lines of the two samples with increasing temperature difference between the gas flow and the surroundings. This indicates that compression of the here employed fibrous insulation results in reorientation of the fibres that is beneficial to the radiation resistance, meaning that by a higher thermal resistance can be achieved at the same insulation mass through compression of an initially lower density material.

Finally, samples $128-18-\mathrm{H}$, with the highest density but less compression than the previously discussed samples, have the highest thermal resistance: $10 \%$ and $20 \%$ larger than that of 128-16-H-4 at low and high engine load respectively, as is shown in Fig. 10. Again, the flow interface and friction factor were similar between these samples, so the cause lies in the insulation. More specifically, the difference must have been in the radiation, because also this insulation density is too low to affect the gas conduction. As already shown, compression increases the radiation resistance and so does an increase in density [10]. The volume reduction was, with $14 \%$, considerably smaller than for 96-20 and yet the thermal resistance increase has roughly doubled, this means that the density or the combination has a larger effect than compression alone.

A future differentiation between the effect of density and compression can be made using a more simple setup that allows more combinations of density and compression, such as the one employed by Daryabeigi et al. [10], since the current work indicates that the overhead gas flow is not of direct influence.

\section{Conclusions}

This article describes an experimental campaign that discerns the influence of different aspects of alternative combustion engine exhaust system walls on the friction factor and thermal resistance. These alternative wall configurations consisted of an outer shell, lined with a porous, fibrous insulation layer that was kept in place using a silica fabric and a steel wire-mesh. The insulation density, the amount of insulation compression and wire-mesh pitch were varied. In the same test, also the total thermal resistance of these configurations was determined, thus including the effect of density and compression. Samples were tested over a range of Reynolds numbers and non-dimensional frequencies relevant to combustion engine exhaust gas flow.

Fig. 10 System thermal resistance of the benchmark sample, 128-16-H-4, and those with both insulation compression and higher density: 128-18-H

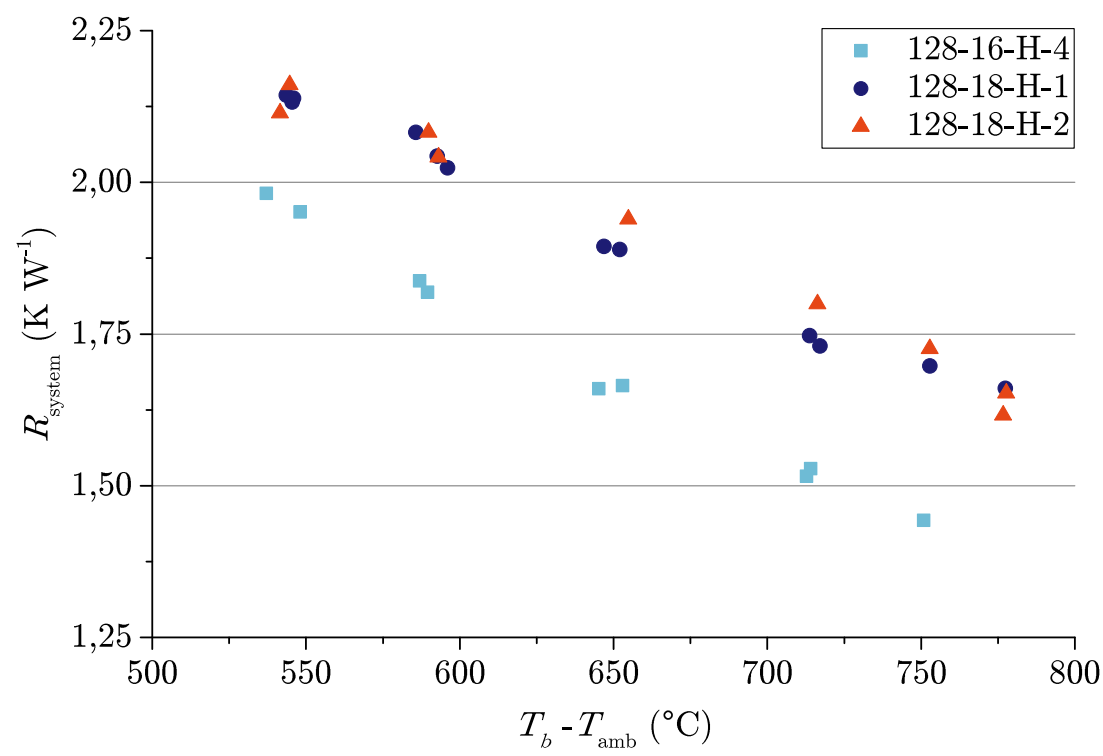


Over the tested range of Reynolds numbers and pulsation frequencies, the friction factor was found constant for the lined sections. Additionally, it did not vary significantly with insulation density/compression. For both wire pitches, the measured friction factors are in line with those reported in literature for similar geometries, but with steady flow and solid walls. Together this indicates that the discrete roughness is the dominant factor and that there is no substantial contribution or interaction of the nonsinusoidally pulsating flow or the compliant wall in between the roughness elements.

The heat loss was determined using a model of the external resistance together with shell temperature measurements. The influence of the fluid-wall interface configuration was in line with that observed for the friction factor. Besides the interface, not only the insulation density, but also the compression of the fibrous insulation substantially increased the radiative heat transfer resistance. It is hypothesized that this happens because the compression leads to insulation fibre reorientation.

Besides the use of this work for alternative exhaust systems and although the investigated configuration is rather specific, its results have further implications. For instance, the observed lack of interaction between wallcompliance, pulsations and discrete roughness within the experimental error could serve as a start for the design of future experiments that involve such potential interactions. Furthermore, given the small number of publications on the friction of compliant walls, this work could add to the general understanding of the phenomenon. Lastly, the effect of fibrous insulation compression opens room for an investigation into the coupling between compression and fibre reorientation.
Acknowledgements The authors wish to express their gratitude for the technical support offered by Dirk Vanderheyden, Bernard Lehaen and Dave Mariën of BOSAL ECS n.v..

\section{Compliance with Ethical Standards}

Conflict of interests On behalf of all authors, the corresponding author states that there is no conflict of interest.

Open Access This article is licensed under a Creative Commons Attribution 4.0 International License, which permits use, sharing, adaptation, distribution and reproduction in any medium or format, as long as you give appropriate credit to the original author(s) and the source, provide a link to the Creative Commons licence, and indicate if changes were made. The images or other third party material in this article are included in the article's Creative Commons licence, unless indicated otherwise in a credit line to the material. If material is not included in the article's Creative Commons licence and your intended use is not permitted by statutory regulation or exceeds the permitted use, you will need to obtain permission directly from the copyright holder. To view a copy of this licence, visit http:// creativecommonshorg/licenses/by/4.0/.

\section{Appendix A: Uncertainty analysis of temperature drop and heat loss rate}

Measurement uncertainty analysis, for instance using the method outlined by Dunn [12], allows differentiation between the contributions of different sources of error. More specifically it provides a framework to estimate the effect of different random and bias errors on the uncertainty of a result. Given the simplicity of the measurement chain for the temperature drop specifically, the uncertainty analysis of the mean using the mentioned method is not too complicated.

The principle of the method is to estimate the true variance of the normal distribution that corresponds to
Fig. 11 The heat loss rate over the instrumented length of the lined section of sample 128-18- $\mathrm{H}-2$ as a function of the temperature difference between the exhaust gas and ambient, based on either the gas flow temperature drop ('internal', solid diamonds), or on the external thermal resistance model ('external', open diamonds) with its forced convection velocity obtained through a least-squares fit of these two rates. Also indicated is the $95 \%$ uncertainty of the heat loss rate based on the gas temperature drop

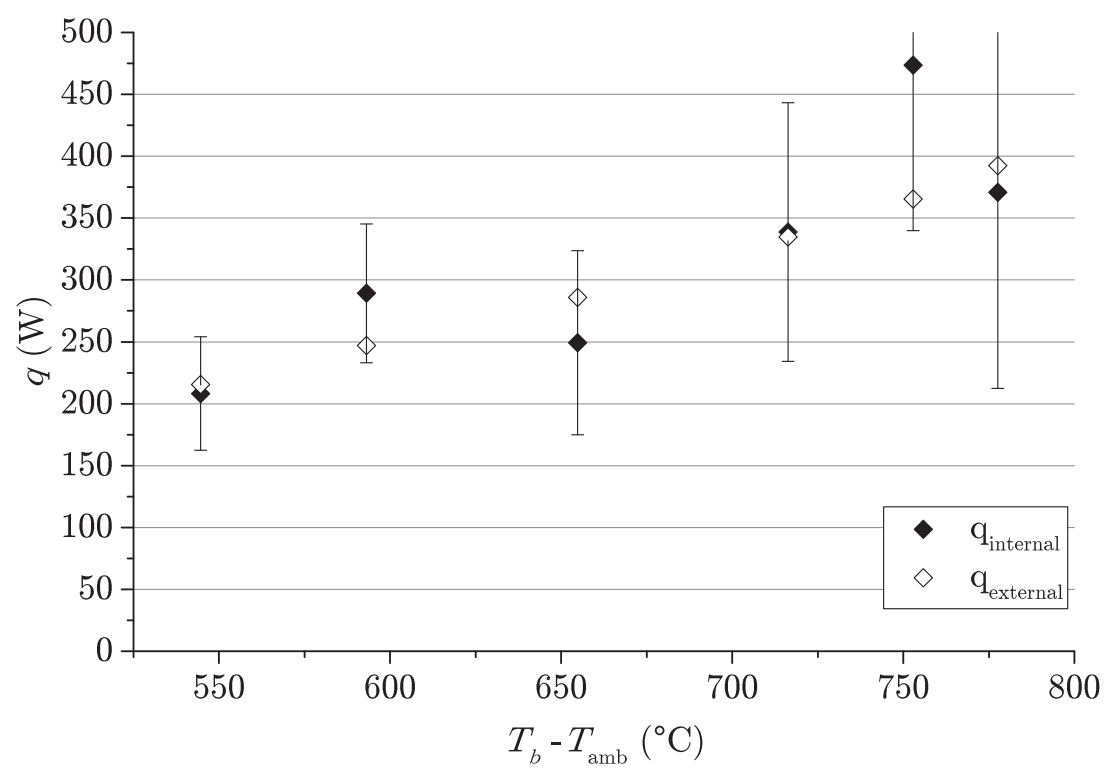


the measurement. As such, it requires an estimate of the variance of the different contributions, even the inherent non-probabilistic ones. The measurement of the temperature drop for a single test stage classifies as a so-called multiple-measurement measurand experiment. Because of the multiple measurements, the standard deviation of the random error is readily available from the data points used for the average temperature drop. The bias (or systematic) error was estimated from comparison with extra tests with the gas temperature drop thermocouples having swapped position. Several tests besides those published here were ran to obtain sufficient information regarding the bias error.

Using the method of Dunn [12] and assuming sufficiently large degrees of freedom to apply the Gaussian distribution results in the following expression for the $95 \%$ confidence uncertainty in the temperature difference

$U_{T, 95} \simeq 2 \sqrt{S_{B_{\mathrm{da}}}^{2}+S_{B_{\mathrm{tc}}}^{2}+S_{\bar{P}_{\mathrm{tc}}}^{2}}$

where $S_{\bar{P}_{\mathrm{tc}}}$ is the standard deviation of the random error in the mean of the thermocouple measurement and $S_{B_{\mathrm{da}}}$ and $S_{B_{\mathrm{tc}}}$ are the standard deviations of the systematic errors corresponding to the data acquisition and thermocouple, respectively.

Quantifying the random and systematic standard deviations is all that remains to establish the uncertainty. The random uncertainty of the mean value can be obtained from the standard deviation of the set of temperature drops used for the average as follows:

$S_{\bar{P}_{\mathrm{tc}}}=\frac{S_{P_{\mathrm{tc}}}}{\sqrt{N}}$

where $N$ is the number of measurements in the set, resulting in $S_{\bar{P}_{\mathrm{tc}}}=0.1{ }^{\circ} \mathrm{C}$. Next to that, the standard deviation of the bias error of the differential temperature measurement was estimated by comparing the difference in the results of a regular measurement of a sample and one with the differential thermocouples swapped. The difference varied between 0.1 and $2.2^{\circ} \mathrm{C}$ for the different stages, leading to a standard deviation of $S_{B_{\mathrm{tc}}}=1.0^{\circ} \mathrm{C}$. Finally, assuming the manufacturer-specified accuracy represents the $95 \%$ confidence bias limit, means that its standard deviation is half the limit: $S_{B_{\mathrm{da}}}=0.1^{\circ} \mathrm{C}$.

Having also established the uncertainty of the specific heat and the mass flow rate through the uncertainty in the correlations and equipment, allows the calculation of the uncertainty of the heat loss rate through the propagation of the relative errors. The resulting $95 \%$ overall uncertainty of the heat loss estimate based on the gas temperature drop is indicated in Fig. 11.

\section{Appendix B: Thermocouple heat balance model}

The thermal interaction of a gas thermocouple with the surrounding sample in the case of the experiment described in this article was modelled to estimate the temperature difference between the gas and that of the different hot junctions along its length. ${ }^{3}$ This was done for both the steel mesh and the solid steel liner (HS) configuration because of the difference in wall roughness and corresponding wall surface temperature.

In general, the equilibrium temperature of a sheathed thermocouple in a gas flow is the result of the balance between different heat transfer mechanisms [31]:

$q_{\mathrm{cond}}=q_{\mathrm{rad}}+q_{\mathrm{conv}}$

where $q_{\text {cond }}$ represents the heat transfer rate through conduction, $q_{\mathrm{rad}}$ is the heat transfer rate through radiation and $q_{\text {conv }}$ is the heat transfer rate through forced convection.

The equilibrium temperature was obtained by solving Eq. 11 numerically for the one-dimensional case of a thermocouple discretized into eight isothermal elements of equal length. The contributions of radiation, convection and conduction were based on the temperature difference between the node in the centre of each element and the object of interaction. The set of elements covers the section of the thermocouple that was exposed to the gas, so the last element ends at the wall.

The conductive heat transfer was estimated as simple one-dimensional conduction, using the average temperature between the two elements as the input for the calculation of the thermal conductivity of Inconel. The contribution of the magnesium oxide and the thermocouple wires inside the individual thermocouples was deemed negligible and thus the elements were given the area and conductivity corresponding to the sheaths. The highly temperaturedependent thermal conductivity of Inconel was taken into account using the following linear approximation as a function of the temperature in Celsius: $\lambda=14.221+$ $0.0162 T$. For the last element, the conductive heat exchange with segment of the thermocouple beyond the flow had to be approximated. This was done by assuming that the length through the insulation was adiabatic and that the thermocouple temperature at the shell, so in the mount, equalled that of the shell.

The nett radiation energy transfer between the two grey bodies of the thermocouple and the duct inner wall, was approximated by that between two concentric pipes with the one having a much larger area than the other: $q_{\mathrm{rad} 12}=$ $\epsilon_{1} \sigma\left(T_{1}^{4}-T_{2}^{4}\right)$. The required hemispherical emissivity of the oxidized Inconel sheath of the thermocouple was estimated

\footnotetext{
${ }^{3}$ See Section 2.3 .
} 
Fig. 12 Gas temperature as a function of distance along the thermocouple from the tip, for both model and thermocouple reading ('tc') for four different stages of the test sequence of 128-16-HS-1. The model uses the measured temperature as input gas temperature

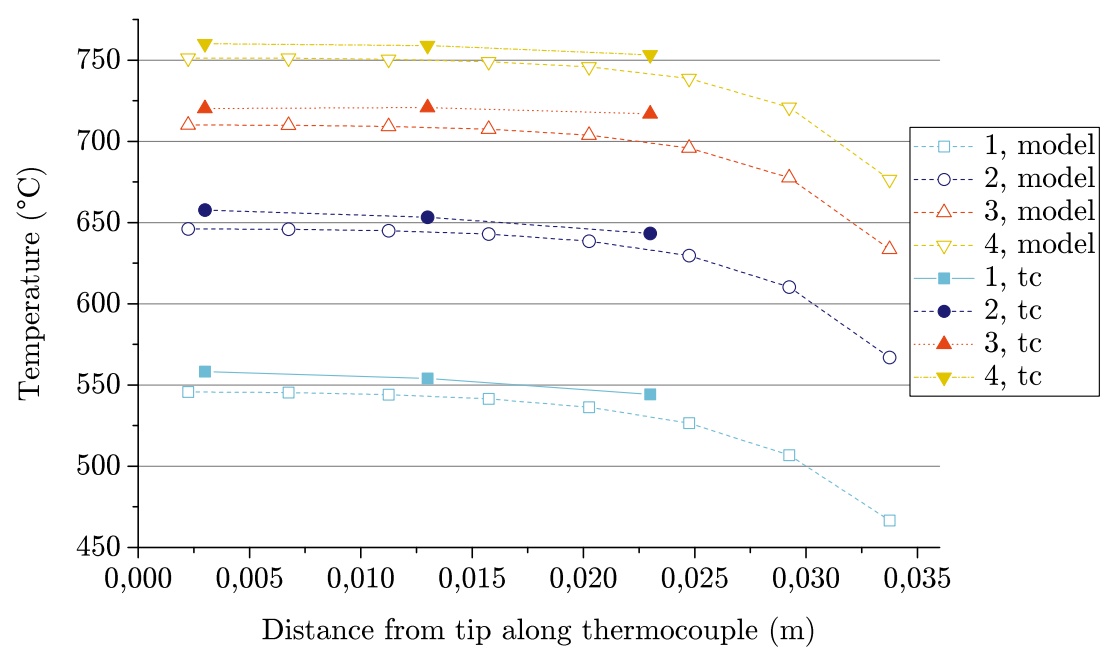

at 0.83 . Next to this, the emissivity of the exhaust gas was calculated according to the method presented in [36] because of the presence of the selective radiators $\mathrm{H}_{2} \mathrm{O}$ and $\mathrm{CO}_{2}$. Its emissivity, over a distance relevant to this model, was less than 0.1 and thus ignored.

The wall temperature of the sample required for the radiation estimate was approximated by solving the combined heat transfer from gas to the wall for the temperature difference needed to have the heat transfer rate found earlier. The convective heat transfer rate for the wire-mesh lined wall was based on the approximation for the Nusselt number from Norris ${ }^{4}$ [30]. Similarly, the Nusselt number for the solid-walled sample was obtained from the expression of Gnielinski found in Table 8.3 of Karwa [20]. The gas radiation in both cases was determined according to [36].

For the thermocouple, the Nusselt number for forced convection over a cylinder in cross-flow was calculated using equations (3-5) of Gnielinski [15], approximating the crosssectional variation in velocity using the velocity defect law [17] and the friction velocity. As a first approximation, the temperature profile was assumed equal to the velocity profile, because the Prandtl number of the flue gas is not too far from unity (0.74) and the thermal boundary layer thus has a thickness similar to that of the momentum one [20]. The temperatures at the extremes of this profile were the wall temperature as estimated for the radiation and the measured gas temperature. The thermal conductivity of the fluid was that according to the bulk fluid temperature.

Using the above estimations for the contributions of conduction, convection and radiation, the temperature distribution along the thermocouple could be estimated for the two main wall configurations. In order to validate

\footnotetext{
${ }^{4}$ Using a coefficient of 0.5 because it matches the wire sources better than the general coefficient of 0.63 .
}

the model, its thermocouple temperature distribution was calculated for four different stages of the performed experiment and compared to the temperatures of the three hot junctions of the middle gas thermocouple during those stages. Figure 12 shows both temperature distributions for sample 128-16-HS-1.

The temperature trends along the thermocouple of the model and measurements are similar and the model thus seems to capture the behaviour sufficiently. The model can thus be used to estimate the error resulting from the assumption that the thermocouple temperature equals the gas temperature. For the thermocouple tip, the first element of the model, the difference is shown in Fig. 13. Besides the smooth-walled sample, the temperature difference of the wire mesh sample 128-16-H-4 is also shown.

The sample 128-16-H-4 has the wire-mesh for support of the lining and the inaccuracy of the Nusselt number and thus the wall temperature itself, is larger than for HS-1. The increased roughness is nevertheless coupled to increased heat transfer, resulting in a higher wall temperature and consequently a smaller temperature difference with the gas. This is reflected in the smaller temperature difference found by the model when compared to the solid smooth wall.

\section{Appendix C: Viscous dissipation}

The heat balance between gas temperature and heat flux to the boundary in the form of Eq. 4 is only valid if viscous dissipation is negligible. The validity of this assumption is worth investigating because of the uncommon combination of a wall with a relative large friction factor, peak fluid velocities of over $50 \mathrm{~m} \mathrm{~s}^{-1}$ and a much larger fluid viscosity than at room temperature.

To estimate the magnitude of viscous dissipation we start from the same principal equation as for the heat 
Fig. 13 Temperature difference between the gas temperature and the sheath temperature according to the proposed model for samples 128-16-HS-1 and 128-16-H-4

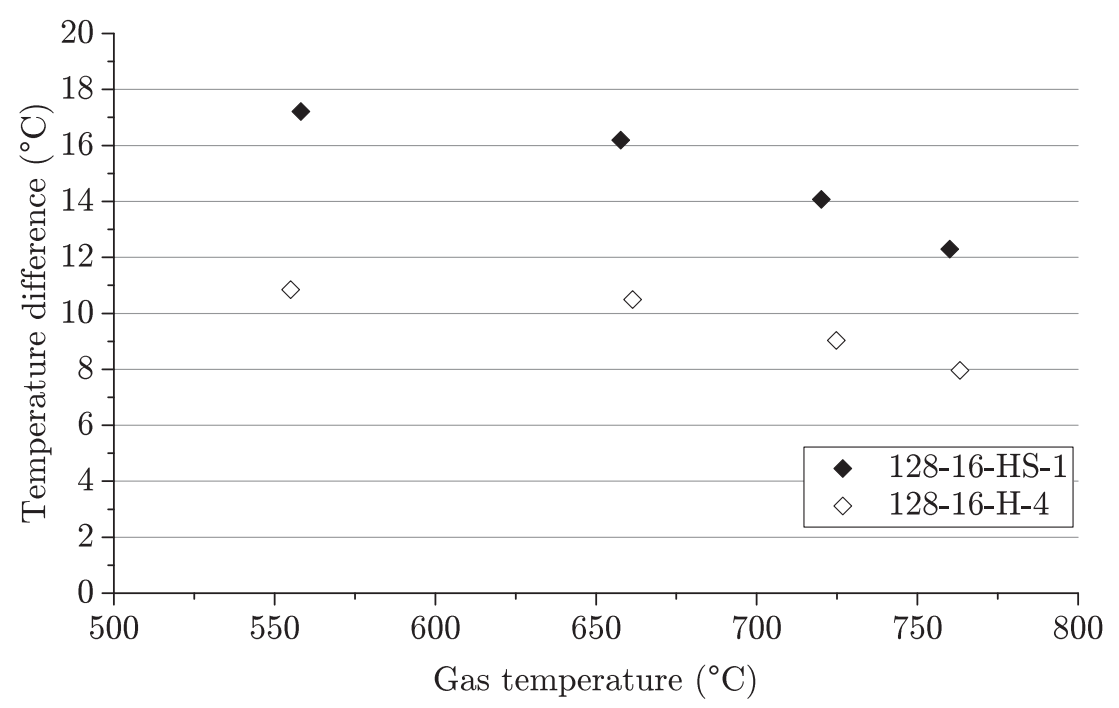

balance, namely the general expression for the thermal energy balance of a differential fluid element [29]:

$$
\begin{aligned}
\rho c_{\mathrm{p}} \frac{\mathrm{D} T}{\mathrm{D} t}= & -\nabla \cdot \mathbf{q}+\beta T \frac{\mathrm{D} p}{\mathrm{D} t} \\
& +\mu\left[-\frac{2}{3}\left(\chi \frac{\mathrm{D} p}{\mathrm{D} t}-\beta \frac{\mathrm{D} T}{\mathrm{D} t}\right)^{2}+\Phi_{\mathrm{inc}}\right]
\end{aligned}
$$

where $\mathbf{q}$ is the heat flux vector, $\beta$ the coefficient of thermal expansion, $\mu$ the dynamic viscosity and $\chi$ the volume compressibility and $\Phi_{\text {inc }}$ the rate of viscous dissipation for an incompressible fluid (unit: $\mathrm{s}^{-2}$ ). This general expression simplifies with further assumptions for the specific case at hand.

Focussing on the time-mean solution and assuming an ideal gas, negligible pressure variations and small enough temperature differences to ignore density variations, but keeping the viscous dissipation term for incompressible fluids reduces Eq. 12 to:

$\rho c_{\mathrm{p}} \overline{\mathbf{u}} \cdot \nabla \bar{T}=-\nabla \cdot \mathbf{q}+\mu \Phi_{\text {inc }}$

where the overscore indicates the time-mean value of the variable.

If we subsequently assume that the total temperature change is a linear combination of that due to viscous dissipation and that resulting from the equilibrium described using Eq. 4, then we can focus on the viscous dissipation only.

Because of the large thermal resistance in the wall and the relatively low conductivity of the gas, it is also assumed that the heat generated through viscous dissipation results predominantly in fluid temperature increase. This means that we can ignore the heat flux term.

The equation still includes the product of the velocity vector and the temperature gradient but there can only be a non-zero time-mean velocity in the streamwise ( $x$ direction. Consequently, the equation further reduces to:

$\rho c_{\mathrm{p}} \bar{u} \frac{\partial \bar{T}}{\partial x}=\mu \Phi_{\text {inc }}$

which is still for a differential fluid element.

Now integrate the expression over the fluid cross-section to obtain the temperature difference over a differential length of the pipe:

$\int \rho c_{\mathrm{p}} \bar{u} \frac{\partial \bar{T}}{\partial x} d A=\int \mu \Phi_{\mathrm{inc}} d A$

which still includes quantities that cannot be compared to those measured during the experiment.

So, we introduce the velocity-weighted bulk temperature $T_{b}$ to be able to express the radially varying temperature in a single number:

$T_{b}=\frac{\frac{1}{\pi R^{2}} \int \bar{u} \bar{T} d A}{\frac{1}{\pi R^{2}} \int \bar{u} d A}$

where the denominator is equal to the bulk velocity $u_{b}$, which varies hardly along the $x$-direction.

The term for viscous dissipation on the right hand side of Eq. 15 also has to be related to measured quantities. In essence, viscous dissipation is the conversion of mechanical energy to heat through viscous heating. In steady-state pipe flow, the loss in mechanical energy can directly be related to the pumping power as follows:

$\int \mu \Phi_{\text {inc }} d A=\int \rho \epsilon d A=\int-\bar{u} \frac{\mathrm{d} \bar{p}}{\mathrm{~d} x} d A$

where $\rho \epsilon$ is the dissipated power per unit volume. 
Fig. 14 Estimate of the temperature increase due to viscous dissipation over the instrumented length versus the temperature difference between the exhaust gas and ambient for two different test sequences of sample 128-16-H-4

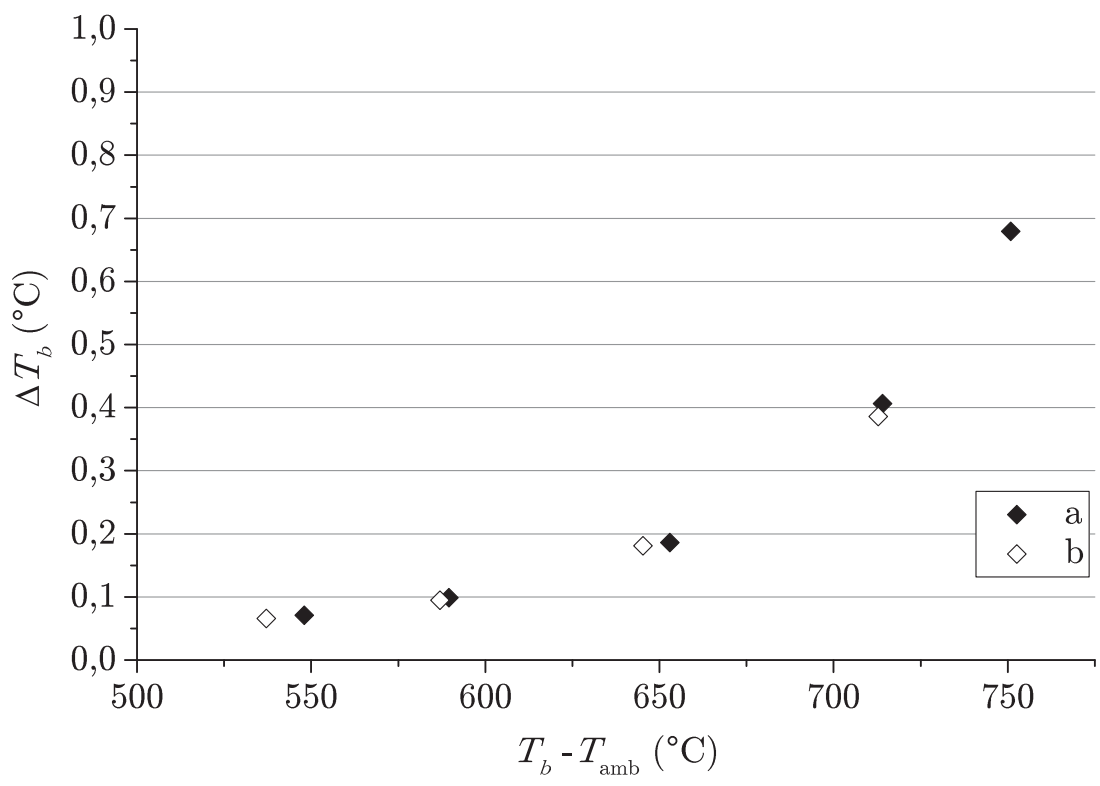

Equating Eqs. 17 and 15, introducing the bulk temperature and performing the integration over the circular cross-section of the sample interior (up to radius $R$ ) yields:

$\rho c_{\mathrm{p}} \pi R^{2} u_{b} \frac{\mathrm{d} T_{b}}{\mathrm{~d} x}=\pi R^{2} u_{b}\left(-\frac{\mathrm{d} \bar{p}}{\mathrm{~d} x}\right)$

which is still for a differential length $d x$. Using the discrete difference over the measurement section rather than the gradient yields:

$\Delta T_{b}=\frac{\Delta p}{\rho c_{\mathrm{p}}}$

where $\Delta p$ is static pressure drop. The outcome of Eq. 19 for sample 12-16-H-4 is shown in Fig. 14. The temperature difference is a good measure for the engine load, note however that not only the gas temperature increases with increasing engine load, but so does the mass flow rate.

The observed temperature differences due to viscous dissipation are negligible compared to the absolute gas temperature, but at the higher engine loads the magnitude is sufficient to require incorporation in Eq. 4 because the measured temperature drop over the sample is of the order of $10^{\circ} \mathrm{C}$. However, this estimated fluid temperature increase due to viscous dissipation could be conservative since part of the pumping power that is now assigned to heating the fluid, in reality went into deforming the fabric and fibres in the wall.

\section{References}

1. ASTM International: ASTM Standard F778 (2014) Standard methods for gas flow resistance testing of filtration media.
2. Bergman TL, Lavine Adrienne S, Incropera FP, Dewitt DP (2011) Fundamentals of heat and mass transfer, 7 th edn. Wiley, New York

3. Berlin P, Dickman O, Larsson F (1992) Effects of heat radiation on carbon/peek, carbon/epoxy and glass/epoxy composites. Composites 23(4):235-243. https://doi.org/10.1016/0010-4361(92)90183-U

4. Bhaganagar K (2008) Direct numerical simulation of unsteady flow in channel with rough walls. Phys Fluids 20(101508):1-15. https://doi.org/10.1063/1.3005859

5. Bhatti MS, Shah RK (1987) Turbulent and transition flow convective heat transfer. In: Kakaç S., Shah R. K., Aung W. (eds) Handbook of single-phase convective heat transfer. Wiley, New York

6. Blair GP (1999) Design and simulation of four-stroke engines. Society of automotive engineers Inc, Warrendale, PA USA

7. Breugem WP, Boersma BJ, Uittenbogaard RE (2006) The influence of wall permeability on turbulent channel flow. J Fluid Mech 562:35-72. https://doi.org/10.1017/S0022112006000887

8. Chandesris M, D'Hueppe A, Mathieu B, Jamet D, Goyeau B (2013) Direct numerical simulation of turbulent heat transfer in a fluid-porous domain. Phys Fluids 25:125110. https://doi.org/10.1063/1.4851416

9. Daryabeigi K (2003) Heat transfer in high-temperature fibrous insulation. J Thermophys Heat Transfer 17(1):10-20. https://doi.org/10.2514/2.6746

10. Daryabeigi K, Cunnington GR, Knutson JR (2011) Combined heat transfer in high-porosity high-temperature fibrous insulation: Theory and experimental validation. J Thermophys Heat Transfer 25(4):536-546. https://doi.org/10.2514/1.T3616

11. Dittus FW, Boelter LMK (1930) Heat transfer in automobile radiators of the tubular type. University of California Publications in Engineering 2(13):443-461. https://doi.org/10.1016/0735-1933(85)90003-X

12. Dunn PF (2010) Measurement and data analysis for engineering and science, 2nd edn. CRC Press, Boca Raton

13. Engineering Sciences Data Unit (ESDU): Losses caused by friction in straight pipes with systematic roughness elements. Tech. Rep. 79014, IHS Markit (2007)

14. European Commission: Commission regulation (EU) 2016/646 of 20 april 2016 amending regulation (EC) no 692/2008 as regards 
emissions from light passenger and commercial vehicles (Euro 6). Official Journal of the European Union (2016). L109

15. Gnielinski VVDI e. V (ed) (2010) G6 heat transfer in crossflow around single tubes, wires, and profiled cylinders, 2nd edn. Springer-Verlag, Heidelberg Berlin. https://doi.org/10.1007/978-3-540-77877-6

16. Hahn S, Je J, Choi H (2002) Direct numerical simulation of turbulent channel flow with permeable walls. J Fluid Mech 450:259-285. https://doi.org/10.1017/S0022112001006437

17. Hinze JO (1975) Turbulence. McGraw-Hill, New York

18. Kabelac S, Vortmeyer D (2010) K1 radiation of surfaces. In: VDI e. V (ed) VDI heat Atlas. 2nd edn. Springer-Verlag, Heidelberg Berlin. https://doi.org/10.1007/978-3-540-77877-6

19. Kakaç S., Shah R. K., Aung W (eds) (1987) Handbook of singlephase convective heat transfer. Wiley, New York

20. Karwa R (2017) Heat and mass transfer. Springer, Singapore. https://doi.org/10.1007/978-981-10-1557-1

21. Kirmse RE (1979) Investigations of pulsating turbulent pipe flow. J Fluids Eng 101(4):436-442. https://doi.org/10.1115/1.3449007

22. Kleiber M, Joh R (2010) D1 calculation methods for thermophysical properties. In: VDI e. V (ed) VDI heat Atlas. 2nd edn. Springer-Verlag, Heidelberg Berlin. https://doi.org/10.1007/978-3-540-77877-6

23. Kleiber M, Joh RVDI e. V (ed) (2010) D3 properties of pure fluid substances, 2nd edn. Springer-Verlag, Heidelberg Berlin. https://doi.org/10.1007/978-3-540-77877-6

24. Kuwata Y, Suga K (2016) Lattice boltzmann direct numerical simulation of interface turbulence over porous and rough walls. Int $\mathrm{J}$ Heat Fluid Flow 61:145-157. https://doi.org/10.1016/j.ijheatfluidflow.2016.03.006

25. Lee SC, Cunnington GR (2000) Conduction and radiation heat transfer in high-porosity fiber thermal insulation. J Thermophys Heat Transfer 14(2):121-136. https://doi.org/10.2514/2.6508

26. Lodahl CR, Sumer BM, Fredsøe J (1998) Turbulent combined oscillatory flow and current in a pipe. J Fluid Mech 373:313-348. https://doi.org/10.1017/S0022112098002559

27. Ludlow JC, Kirwan DJ, Gainer JL (1980) Heat transfer with pulsating flow. Chem Eng Commun 7(4-5):211-218. https://doi.org/10.1080/00986448008912559

28. Meng X, de Jong W, Kudra T (2016) A state-of-the-art review of pulse combustion: Principles, modeling, applications and r\&d issues. Renew Sustain Energy Rev 55:73-114. https://doi.org/10.1016/j.rser.2015.10.110
29. Morini GL (2015) Viscous dissipation. In: Li D (ed) Encyclopedia of Microfluidics and Nanofluidics. 2nd edn. Springer, Berlin, pp 3442-3453. https://doi.org/10.1007/978-1-4614-5491-5

30. Norris RH (1970) Some simple approximate heat-transfer correlations for turbulent flow in ducts with rough surfaces. In: Bergles A, Webb R (eds) Augmentation of convective heat and mass transfer. The American Society of Mechanical Engineers, New York, pp 16-26

31. Park RM, Carroll RM, Bliss P, Burns GW, Desmaris RR, Hall FB, Herzkovitz MB, MacKenzie D, McGuire EF, Reed RP, Sparks LL, Wang TP (1993) Manual on the use of thermocouples in temperature measurement. 4th edn. ASTM International, West Conshohocken. https://doi.org/10.1520/MNL12-4TH-EB. MNL12-4TH

32. Picard A, Davis RS, Gläser M., Fujii K (2008) Revised formula for the density of moist air (CIPM-2007). Metrologica 45:149-155. https://doi.org/10.1088/0026-1394/45/2/004

33. Sheriff N, Gumley P (1966) Heat transfer and friction properties of surfaces with discrete roughnesses. Int J Heat Mass Transf 9(12):1297-1320. https://doi.org/10.1016/0017-9310(66)90130-X

34. Siuru Jr WD, Logan Jr E (1977) Response of a turbulent pipe flow to a change in roughness. J Fluids Eng 99(3):548-553. https://doi.org/10.1115/1.3448842

35. Timoshenko SP, Goodier JN (1987) Theory of elasticity, 3rd edn. McGraw-Hill, New York

36. Vortmeyer D, Kabelac S (2010) K3 gas radiation: Radiation from gas mixtures. In: VDI e. V (ed) VDI heat Atlas. 2nd edn. Springer-Verlag, Berlin Heidelberg. https://doi.org/10.1007/978-3-540-77877-6

37. Wendland DW (1993) Automobile exhaust-system steady-state heat transfer. SAE Technical Paper 931085. https://doi.org/10.4271/931085

38. Xia QJ, Huang WX, Xu CX (2017) Direct numerical simulation of turbulent boundary layer over a compliant wall. J Fluids Struct 71:126-142. https://doi.org/10.1016/j.jfluidstructs.2017.03.005

39. Zhao S, Zhang B, He X (2009) Temperature and pressure dependent effective thermal conductivity of fibrous insulation. Int $\mathrm{J}$ Thermal Sci 48(2):440-448. https://doi.org/10.1016/j.ijthermalsci.2008.05.003

Publisher's note Springer Nature remains neutral with regard to jurisdictional claims in published maps and institutional affiliations. 